\title{
Egyptian 2/D Table (D Prime Number): An Entirely New Analysis Consistent with the Idea of a Progressive Teamwork
}

\author{
Lionel Bréhamet \\ Independent Scholar, Bordeaux, France
}

Email address:

brehamet.1@orange.fr

\section{To cite this article:}

Lionel Bréhamet. Egyptian 2/D Table (D Prime Number): An Entirely New Analysis Consistent with the Idea of a Progressive Teamwork. History Research. Vol. 5, No. 2, 2017, pp. 17-29. doi: 10.11648/j.history.20170502.11

Received: October 8, 2016; Accepted: February 17, 2017; Published: April 1, 2017

\begin{abstract}
For $h=3$ or 4 , Egyptian decompositions into $h$ unit fractions, like $2 / D=1 / D_{l}+\ldots+1 / D_{h}$, were given by using $(h-l)$ divisors $\left(d_{i}\right)$ of $D_{l}$. This ancient modus operandi, well recognized today, provides $D_{i}=D D_{l} / d_{i}$ for $i$ greater than 1 . Decompositions selected (depending on $d_{i}$ ) have generally been studied by modern researchers through the intrinsic features of $d_{i}$ itself. An unconventional method is presented here without considering the $d_{i}$ properties but just the differences $d_{h-I^{-}} d_{h}$. In contrast to widespread ideas about the last denominator like ' $D_{h}$ smaller than 1000 ', it is more appropriate to adopt a global boundary of the

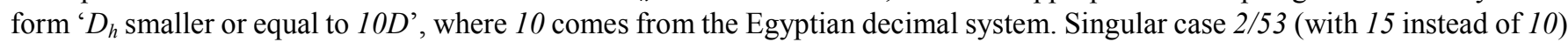
is explained. The number of preliminary alternatives before the final decisions is found to be so low (71) for $h=3$ or 4 that a detailed overview was possible in the past. A simple additive method of trials, independent of any context, can be carried out, namely $2 n+1=d_{2}+\ldots+d_{h}$. Clearly the decisions fit with a minimal value of the differences $d_{h-1^{-}} d_{h}$, independently of any $d_{i}$ values.
\end{abstract}

Keywords: Rhind Papyrus, 2/n Table, Egyptian Fractions

\section{Introduction}

The recto of the Rhind Mathematical Papyrus (RMP) $[1,2$, $3]$ contains the so-called Egyptian $2 / n$ table, written in Hieratic The genesis of a project such as build this table will never really discovered. This is not a project as impressive as the construction of a pyramid or temple, however it has been well and truly succeeded. It is impossible to doubt that pyramid works have not been carried out without a hierarchy of teams well organized in various specialties. A perfectly organized hierarchy that included team leaders and supervisors.

It is not hard to imagine that a structured similar organization was also used for the table [renamed as 2/D table throughout our paper]. This table has not been an exercise in style. It is imperative to keep in mind that it can not be the work of a single scribe, but surely results of indefinite periods of trials and improvements done by an elite team of scribes talented for calculating. As it is well known through dialogues of Plato, the idea of a small number of scholars (philosophers) comes frequently. To these people only, was reserved the right to reflect on issues such as calculations or the study of numbers. He knew very well that this type of elite was present in the community of scribes of ancient Egypt. He was also aware of their very advanced knowledges in these areas, but without knowing all secrets. There is no reason today to reject the idea of an elite team or even a chief scribe empowered to decide the last.

The time for carrying the table was perhaps over more than a generation ${ }^{1}$ in order to provide a satisfactory completed product. In such a product nothing should have been left to chance and everything has been deliberately chosen. This is not like a school exercise where one can use a decomposition rather than another to solve a given problem.

Once found suitable methods for calculations, it becomes possible to take a look at "the preliminary draft" in its entirety. This look is necessary in order to preserve an overall

\footnotetext{
${ }^{1}$ The creative flash of an inspired scholar (ancient or modern) is short. What is generally much longer is the development of the idea and achievement of tools (theoretical or practical) necessary for its application. Of course once the tools lapped their use takes little time!
} 
coherence. Some difficulties thus may be highlighted and resolved by a minimum of general decisions, the simplest as possible. The number of potential solutions appears as considerably lower than $a b$ initio unrealistic calculations published in the modern literature [4, 5], namely 22295 or around 28000. We find that it is enough to consider only $71+71$ possibilities, then results could be examined before making consistent decisions. This is realistic. A team spirit is very suitable to make obvious the need for a classification and successive resolutions of difficulties encountered during the project progress. Directives given by a leader are implied. All these ideas have put us on the track to a comprehensive approach. These ones are the filigree of our analysis. In fact, this paper is an attempt to change our paradigm by a shift [6] back toward the past. It is also fully in line to the criticism of Zahrt [7] about some erroneous ideas on the simplistic nature of ancient Egyptian mathematics.

\section{Data from the Papyrus}

RMP is also well known by the name of his transcriber, the scribe Ahmes. According to Lefebvre [8], his hieroglyphic name looks like - $\pitchfork \Gamma$ e with standard translitteration $\boldsymbol{\Gamma} \boldsymbol{h}-\boldsymbol{m} \boldsymbol{s} \boldsymbol{w}$. This latter copied the document around 1650 BCE. The source, now lost, could date from XIIth dynasty, a golden age of the middle kingdom. RMP recto shows a table of 2 divided by numbers $D$ from 5 up to 10 into "unit fractions". Number 3 may be considered as implicitly included, because its decomposition is used in the verso for some problems or it appears elsewhere in Papyrus Kahun [9]. This fact has been commented pertinently by Abdulaziz [10].

For $D$ prime only (except number101), we present below a reordered excerpt from the $2 / D$ table by using as subscripts our favorite numbers $m$, that just show the multiplicity of a denominator with $D$. Please note that they are not the red auxiliary numbers used by Ahmes, ie those "decoded" by Gardner [11], but related with these latter by means of the divisors of the first denominator $D_{l}$.

Table 1. Reordered $2 / D$ table for prime numbers $D$

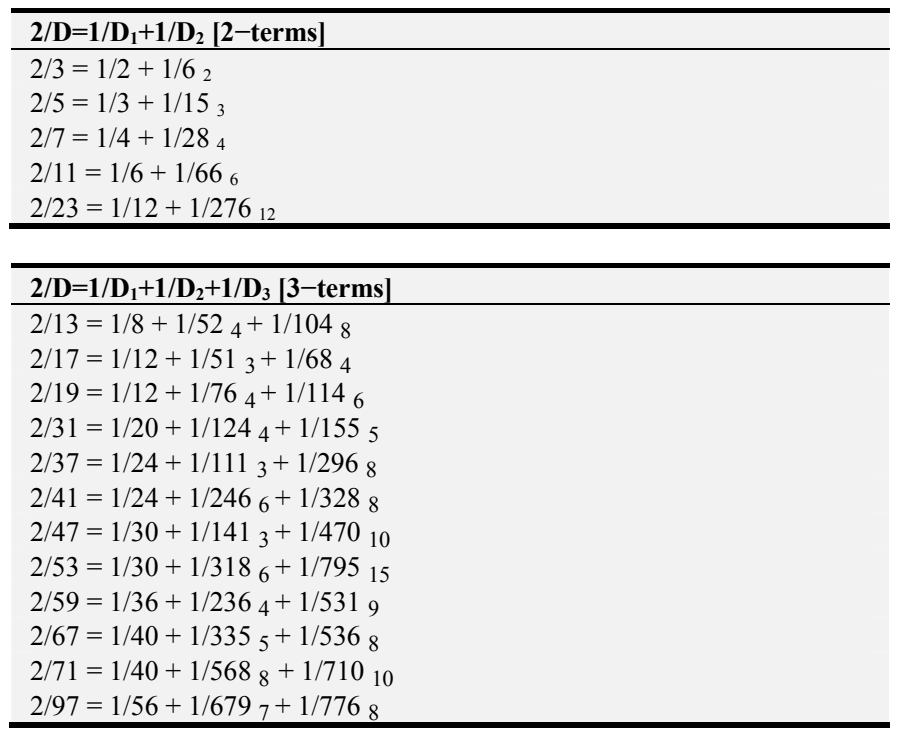

\begin{tabular}{l}
\hline $\mathbf{2 / D}=\mathbf{1} / \mathbf{D}_{\mathbf{1}}+\mathbf{1} / \mathbf{D}_{\mathbf{2}}+\mathbf{1} / \mathbf{D}_{3}+\mathbf{1} / \mathbf{D}_{\mathbf{4}}[\mathbf{4}$-terms] \\
\hline $2 / 29=1 / 24+1 / 58_{2}+1 / 174_{6}+1 / 232_{8}$ \\
$2 / 43=1 / 42+1 / 86_{2}+1 / 129_{3}+1 / 301_{7}$ \\
$2 / 61=1 / 40+1 / 244_{4}+1 / 488_{8}+1 / 610_{10}$ \\
$2 / 73=1 / 60+1 / 219_{3}+1 / 292_{4}+1 / 365_{5}$ \\
$2 / 79=1 / 60+1 / 237_{3}+1 / 316_{4}+1 / 790_{10}$ \\
$2 / 83=1 / 60+1 / 332_{4}+1 / 415_{5}+1 / 498_{6}$ \\
$2 / 89=1 / 60+1 / 356_{4}+1 / 534_{6}+1 / 890_{10}$ \\
\hline
\end{tabular}

\section{Outlines of a Global Approach}

Actually the whole $2 / D$ project can been viewed as a 3-component set.

FIRST OPERATION: discovery of a unique [2-terms] solution, if $D$ is a prime number.

SECOND OPERATION: for a sub-project [composite numbers] from 9 up to 99 , realize that a mini-table, with just four numbers, enables to derive all the composite numbers by using a multiplicative operation ${ }^{2}$.

Four numbers, 3, 5, 7, 11 are enough. For instance 99 is reached with $3 \times 33$ or $11 \times 9$.

This mini-table, a kind of 'Mother-table', looks as follows:

Table 2. Basic Mother-Table.

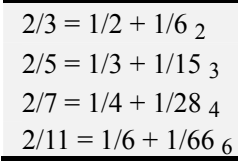

One sees the first four two-terms decompositions of $2 / D . D$ being prime, the table is unique.

In 'theory', except if a better decision should be token, any fraction $2 / D$ ( $D$ composite) could be decomposed from this table by dividing a given row by a convenient number. Consider an example

$2 / 65=[($ row 2$) /($ number 13$)]=1 / 39+1 / 195_{3}$, which is the solution adopted in the papyrus. As a matter of fact, all decompositions for the sub-project were given in two-terms (except for 2/95 as a logical consequence of the guidelines adopted by the scribes, that we will justify properly later) ${ }^{3}$.

As the 'Mother-table' has no need to higher value than 11 for the sub-project, we can better understand that, from 13, it could have been decided to leave decompositions into 2 terms.

THIRD OPERATION: nothing does more obstacle to start a main part of the whole project, namely decompositions into 3 (or 4 terms if necessary), for all prime numbers starting from 13 until 97.

The study carried in this paper is devoted to the third phase.

\section{General Presentation}

We could have present the problems in the Egyptian manner, as did Abdulaziz [10] like for example

\section{$47 \overline{30} \overline{141} \overline{470}$}

\footnotetext{
${ }^{2}$ Idea already suggested by Gillings [4]

3 All the Egyptian decompositions for composite numbers are analyzed in our second paper [12]
} 
which means $2 / 47=1 / 30+1 / 141+1 / 470$, but we preferred a modern way, more understandable to us today. This is unrelated to the spirit in which we thought.

Consider $D_{l}$ as given ( $D_{l}$ is an unknown value to be found). Assume now that $d_{2}, d_{3}, d_{4}$ are distinct divisors of $D_{1}$, with $d_{2}>$ $d_{3}>d_{4}$. These numbers are also unknowns to find.

In order to standardize the notations, $D$ is used for Denominators and $d$ for divisors.

Look at the following (modern) equations that decompose the 'unity' in 3 or 4 parts:

$$
\begin{gathered}
1=\mathrm{D} / 2 \mathrm{D} 1+\mathrm{d} 2 / 2 \mathrm{D} 1+\mathrm{d} 3 / 2 \mathrm{D} 1, \\
1=\mathrm{D} / 2 \mathrm{D} 1+\mathrm{d} 2 / 2 \mathrm{D} 1+\mathrm{d} 3 / 2 \mathrm{D} 1+\mathrm{d} 4 / 2 \mathrm{D} 1 .
\end{gathered}
$$

It can be viewed under another standpoint like additive operations on integers:

$$
\begin{aligned}
& 2 D_{I}=D+d_{2}+d_{3}, \\
& 2 D_{I}=D+d_{2}+d_{3}+d_{4} .
\end{aligned}
$$

Since $d_{2}, d_{3}, d_{4}$ divide $D_{1}$ then we are sure to find Egyptian decompositions. Indeed, dividing by $D D_{l}$ we always get sums of unit fractions:

$$
\begin{gathered}
2 / D=1 / D_{I}+1 /\left[\left(D_{l} / d_{2}\right) D\right]+1 /\left[\left(D_{l} / d_{3}\right) D\right] . \\
\left.2 / D=1 / D_{I}+1 /\left[\left(D_{l} / d_{2}\right) D\right]+1 /\left[\left(D_{l} / d_{3}\right) D\right]\right]+1 /\left[\left(D_{l} / d_{4}\right) D\right] .
\end{gathered}
$$

This method was apparently followed [11] in RMP table for prime numbers $D$ from 13 up to 97.

As can be seen, except $D_{l}$, all denominators of each equation appear as a multiple of $D$, namely

$$
D_{i}=m_{i} D \text { or } m_{i}=\left(D / d_{i}\right) \text {. }
$$

Let us briefly summarize the possibilities as follows

$$
\begin{gathered}
2 / D=1 / D_{1}+1 / D_{2}+1 / D_{3} . \\
2 / D=1 / D_{1}+1 / D_{2}+1 / D_{3}+1 / D_{4} .
\end{gathered}
$$

The main task consists in the determination of $D_{l}$ and the convenient choice of $d_{i}$, from the additive equations (3) or (4). The $d_{i}$ 's are the red auxiliary numbers used by the scribe Ahmes.

$$
d_{i}=D_{I} / m_{i}
$$

\section{4. [2-Terms] Analysis}

$$
2 / D=1 / D_{1}+1 / D_{2} \text {. }
$$

The only comment (admirative) on the subject is that the scribes actually found the right solution (unique) to the problem, namely

$$
D_{l}=(D+1) / 2 \text { and } D_{2}=D(D+1) / 2 \text {. }
$$

\section{5. [3-Terms] Analysis}

Right now consider the [3-terms] cases. Egyptians gave:
Table 3. [3-terms] with $2 D_{1}$ decomposition

\begin{tabular}{lll}
\hline Ahmes's selections [3-terms] & $\Leftarrow$ & Unity decomposition \\
\hline $2 / 13=1 / 8+1 / 52_{4}+1 / 104_{8}$ & $16=13+2+1$ \\
$2 / 17=1 / 12+1 / 51_{3}+1 / 68_{4}$ & $24=17+4+3$ \\
$2 / 19=1 / 12+1 / 76_{4}+1 / 114_{6}$ & $24=19+3+2$ \\
$2 / 31=1 / 20+1 / 124_{4}+1 / 155_{5}$ & & $40=31+5+4$ \\
$2 / 37=1 / 24+1 / 111_{3}+1 / 296_{8}$ & & $48=37+8+3$ \\
$2 / 41=1 / 24+1 / 246_{6}+1 / 328_{8}$ & $\Leftarrow$ & $48=41+4+3$ \\
$2 / 47=1 / 30+1 / 141_{3}+1 / 470_{10}$ & & $60=47+10+3$ \\
$2 / 53=1 / 30+1 / 318_{6}+1 / 795_{15}$ & & $60=53+5+2$ \\
$2 / 59=1 / 36+1 / 236_{4}+1 / 531_{9}$ & $72=59+9+4$ \\
$2 / 67=1 / 40+1 / 35_{5}+1 / 536_{8}$ & $80=67+8+5$ \\
$2 / 71=1 / 40+1 / 568_{8}+1 / 710_{10}$ & $80=71+5+4$ \\
$2 / 97=1 / 56+1 / 679_{7}+1 / 776_{8}$ & $112=97+8+7$ \\
\hline
\end{tabular}

The task of finding $D_{1}$ is rather simple, from the moment when one realizes that it is enough to establish a table of odd numbers $(2 \mathrm{n}+1) \mid \mathrm{n} \geq 1$ as a sum of two numbers $d_{2}+d_{3}$, with $d_{2}>d_{3}$. This is easy to do and independent of any context. The table contains $\mathrm{n}$ doublets $\left\{d_{2}, d_{3}\right\}$ and sup $\left(d_{2}\right)=2 \mathrm{n}$. One can start with the lowest values as follows: $d_{3}=1, d_{2}=2,4,6, \cdots ; d_{3}=2$, $d_{2}=3,5,7, \cdots$ and so on.

From (4) the first candidate possible for $D_{1}$ starts at an initial value $D_{1}{ }^{0}=(D+1) / 2$ as in Fibonnaci's studies [13]. We can search for general solutions of the form

$$
\begin{gathered}
D_{1}{ }^{n}=D_{1}{ }^{0}+n, \\
2 D_{1}{ }^{\mathrm{n}}-\mathrm{D}=2 n+1=d_{2}+d_{3} .
\end{gathered}
$$

Since one of the two $D_{1}$ divisors $\left\{d_{2}, d_{3}\right\}$ is even, then $D_{1}$ can not be odd, it must be even. This was rightly stressed by Bruins [11]. From the first table of doublets, a new table (of trials) is built, where this time doublets are selected if $d_{2}, d_{3}$ divide $\left(D+d_{2}+d_{3}\right) / 2$. This provides a $D_{1}{ }^{n}$ possible. In this favorable case, first $D_{3}$ is calculated by $D D_{1} / d_{3}$, then $D_{2}$ by $D D_{1} / d_{2}$.

For $D$ given, the table of trials defined by the equation just below

$$
2 n+1=d_{2}+d_{3}, \text { where } \mathrm{d}_{2} \text { and } \mathrm{d}_{3} \text { divide } \mathrm{D}_{1}{ }^{\mathrm{n}},
$$

is bounded by a $n_{\max }{ }^{4}$. By simplicity in our tables, $D_{l}{ }^{n}$ will not be written as $D_{l}^{n}\left(d_{2}, d_{3}\right)$.

Even by hand, a realization of this table takes few time. It is the same if the Egyptian addition is used.

For example decompositions into 3 terms lead to a total of trials with only 71 possibilities! From this low value, it is conceivable to present all results according to an appropriate parameter. Once found a $d_{3}$, a good idea would be select a $d_{2}$ the closest as possible of $d_{3}$. This provides a type of classification never glimpsed to our knowledge. Thus, a key parameter of our paper is defined as follows:

$$
\Delta_{d}=d_{2}-d_{3}
$$

Remarks: Clearly (15) is related to Bruins's method of "parts" redistribution $d_{2}, d_{3}$ [14]. However our method is 'artisanal' and does not need to know the arithmetic properties of $D_{l}$. Once $D$ given, $D_{l}$ are found by trials, without

\footnotetext{
${ }^{4}$ It can be proved that no solution can be found beyond $n=(D-3) / 2$.
} 
calculations. Unlike to Bruins which sought some forms of $D_{l}$ for finding then possible $D$ values. The approach is quite different as well as the reasons justifying the Egyptian choices.

Although our conceptual formalism is different from that of Abdulaziz [9], we (fortunately) found some similarities, but also elements without counterpart to us. A welcome unison is the following:

Let us consider its fractional parameter $[R]$ that is crucial for all its analyses. In our notations:

$$
D_{l}[R]=\left(2 D_{l}-D\right)=2 n+1=d_{2}+d_{3},
$$

or equivalently expressed

$$
[R]=1 /\left[\left(D_{l} / d_{2}\right)\right]+1 /\left[\left(D_{l} / d_{3}\right)\right] .
$$

When it is said "... keeping the terms of $[R]$, less than 10 was an essential part of determining how $2: n$ is to be decomposed.", this should be understood as $\left(D_{I} / d_{3}\right) \leq 10$ and formulated for us as the condition (20) with a Top-flag $\mathrm{T}_{f}{ }^{[3]}$ $=10$. (See below for our Top-flag definition)

However note that the 'necessity' of our Top-flag comes directly from the value of $D$, without constituting a check on $D_{l}$. That only follows from (19), see below.

In contrast, parameter [Q], defined in Ref. [10] by [Q] = 1$[R]$, does not appear to us and plays no role in our analyses. In addition, as the impact of closeness $\left(\Delta_{d}\right)$ does not seem to have been apprehended, it is clear that our argumentation will generally be different. Even if, for some 'easy' cases, we agree.

In short, for producing their final table, we assume that the scribes have analyzed all preliminary trial results before doing their choice among various alternatives, considered in their totality, not individually.

Furthermore, due to decimal numeration used by ancient Egyptians, one can easily understand that a boundary with a Top-flag $\mathrm{T}_{f}{ }^{[3]}$ for the last denominator was chosen with a priority value equal to 10 (if possible according to the results given by trials).

The idea of a Top-flag is far to be a 'deus ex machina'. It naturally arises if we try to solve the problem of decomposition in full generality. See Appendix A for more details.

Chief scribe wisely decided to impose a upper bound to all the denominators $D_{3}$, such that

$$
D_{3} \leq D \mathbf{T}_{f}^{[3]} \text {. }
$$

As the Egyptian multiplication ( $D$ to be multiplied by $\mathbf{T}_{f}{ }^{[3]}$ ) is very simple, this remains realistic.

This cut-off beyond $\mathbf{T}_{f}{ }^{[3]}$ is equivalent to a mathematical condition on $D_{l}$ :

$$
D_{l} \leq d_{3} \mathbf{T}_{f}^{[3]} \text {. }
$$

Remark that this condition might be exploited from the beginning of the calculations for avoiding to handle too large denominators $D_{3}$. Simply find $d_{3}$, find $d_{2}$, then calculate $D_{1}$, if condition (20) is not fulfilled then quit, do not calculate $D_{3}, D_{2}$ and go to next values for $d_{3}, d_{2}, D_{1}$ and so on.

Actually, if we follow the method of trials for finding the good choices in the order $d_{3 \rightarrow} d_{2 \rightarrow} D_{1}$, we are naturally led to be careful of the closeness of $d_{3}, d_{2}$, measured by $\Delta_{d}$. This can suggest the idea of a classification according to increasing values of $\Delta_{d}$.

Since this classification seriously enlightens many solutions chosen by the scribes, it is not impossible to imagine that this 'artisan method' was actually followed. This is a plausible hypothesis, valueless of evidence obviously. An advantage is also that a similar classification can be applied to the decompositions into 4 terms with the same success, see Sect. 6.

The symbol $E g$ will be used for indicating Egyptian selections in our tables.

\begin{tabular}{|c|c|c|c|c|c|c|}
\hline $\mathbf{n}$ & $2 n+1$ & $\mathbf{d}_{2}$ & $d_{3}$ & $\Delta_{\mathrm{d}}$ & $D^{\mathrm{n}}{ }_{1}$ & Possible [3-terms] decompositions \\
\hline 1 & 3 & 2 & 1 & 1 & 8 & $2 / 13=1 / 8+1 / 52_{4}+1 / 104_{8}^{E g}$ \\
\hline 1 & 3 & 2 & 1 & 1 & 10 & $2 / 17_{a}=1 / 10+1 / 85_{5}+1 / 170_{10}$ \\
\hline 3 & 7 & 4 & 3 & 1 & 12 & $2 / 17_{\mathrm{b}}=1 / 12+1 / 51_{3}+1 / 68_{4}{ }^{E g}$ \\
\hline 2 & 5 & 3 & 2 & 1 & 12 & $2 / 19=1 / 12+1 / 76_{4}+1 / 114{ }^{E g}$ \\
\hline 1 & 3 & 2 & 1 & 1 & 16 & $2 / 29=1 / 16+1 / 232_{8}+1 / 46416$ \\
\hline 2 & 5 & 3 & 2 & 1 & 18 & $2 / 31_{\mathrm{a}}=1 / 18+1 / 186_{6}+1 / 279_{9}$ \\
\hline 4 & 9 & 5 & 4 & 1 & 20 & $2 / 31_{b}=1 / 20+1 / 1244_{4}+1 / 1555_{5}^{E g}$ \\
\hline 1 & 3 & 2 & 1 & 1 & 20 & $2 / 37=1 / 20+1 / 370_{10}+1 / 740_{20}$ \\
\hline 1 & 3 & 2 & 1 & 1 & 22 & $2 / 41_{\mathrm{a}}=1 / 22+1 / 451_{11}+1 / 90222$ \\
\hline 3 & 7 & 4 & 3 & 1 & 24 & $2 / 41_{\mathrm{b}}=1 / 24+1 / 246_{6}+1 / 3288^{E g}$ \\
\hline 2 & 5 & 3 & 2 & 1 & 24 & $2 / 43=1 / 24+1 / 344_{8}+1 / 516_{12}$ \\
\hline 1 & 3 & 2 & 1 & 1 & 28 & $2 / 53=1 / 28+1 / 74214+1 / 148428$ \\
\hline 1 & 3 & 2 & 1 & 1 & 32 & $2 / 61=1 / 32+1 / 976_{16}+1 / 195232$ \\
\hline 2 & 5 & 3 & 2 & 1 & 36 & $2 / 67=1 / 36+1 / 804_{12}+1 / 1206_{18}$ \\
\hline 4 & 9 & 5 & 4 & 1 & 40 & $2 / 71 \mathrm{a}=1 / 40+1 / 5688_{8}+1 / 710_{10}{ }^{E g}$ \\
\hline 6 & 13 & 7 & 6 & 1 & 42 & $2 / 71 b=1 / 42+1 / 426_{6}+1 / 4977$ \\
\hline 1 & 3 & 2 & 1 & 1 & 38 & $2 / 73=1 / 38+1 / 138719+1 / 227438$ \\
\hline 2 & 5 & 3 & 2 & 1 & 42 & $2 / 79=1 / 42+1 / 1106_{14}+1 / 1_{21}$ \\
\hline 1 & 3 & 2 & 1 & 1 & 46 & $2 / 89 a=1 / 46+1 / 204723+1 / 409446$ \\
\hline 3 & 7 & 4 & 3 & 1 & 48 & $2 / 89_{b}=1 / 48+1 / 1068_{12}+1 / 142416$ \\
\hline
\end{tabular}

We can now display a preliminary table of trials, see Table 4.

Table 4. Table of trials [3-terms] with increasing order of $\Delta_{d}$, only 71 possibilities. 


\begin{tabular}{|c|c|c|c|c|c|c|}
\hline n & $2 n+1$ & $\mathbf{d}_{2}$ & $d_{3}$ & $\Delta_{\mathrm{d}}$ & $D^{\mathrm{n}}{ }_{1}$ & Possible [3-terms] decompositions \\
\hline 1 & 3 & 2 & 1 & 1 & 50 & $2 / 97_{a}=1 / 50+1 / 242525+1 / 485050$ \\
\hline 7 & 15 & 8 & 7 & 1 & 56 & $2 / 97_{\mathrm{b}}=1 / 56+1 / 679_{7}+1 / 776_{8}{ }^{E g}$ \\
\hline 3 & 7 & 5 & 2 & 3 & 10 & $2 / 13=1 / 10+1 / 26_{2}+1 / 65_{5}$ \\
\hline 2 & 5 & 4 & 1 & 3 & 12 & $2 / 19=1 / 12+1 / 57_{3}+1 / 228_{12}$ \\
\hline 2 & 5 & 4 & 1 & 3 & 24 & $2 / 43=1 / 24+1 / 258_{6}+1 / 103224$ \\
\hline 3 & 7 & 5 & 2 & 3 & 30 & $2 / 53=1 / 30+1 / 318_{6}+1 / 79515^{E g}$ \\
\hline 2 & 5 & 4 & 1 & 3 & 32 & $2 / 59=1 / 32+1 / 4728+1 / 188832$ \\
\hline 2 & 5 & 4 & 1 & 3 & 36 & $2 / 67_{a}=1 / 36+1 / 6039+1 / 241236$ \\
\hline 6 & 13 & 8 & 5 & 3 & 40 & $2 / 67_{\mathrm{b}}=1 / 40+1 / 335_{5}+1 / 536_{8} E g$ \\
\hline 3 & 7 & 5 & 2 & 3 & 40 & $2 / 73=1 / 40+1 / 1584_{8}+1 / 1460_{20}$ \\
\hline 2 & 5 & 4 & 1 & 3 & 44 & $2 / 83=1 / 44+1 / 91311+1 / 365244$ \\
\hline 3 & 7 & 6 & 1 & 5 & 12 & $2 / 17=1 / 12+1 / 34_{2}+1 / 204_{12}$ \\
\hline 4 & 9 & 7 & 2 & 5 & 14 & $2 / 19=1 / 14+1 / 38_{2}+1 / 133_{7}$ \\
\hline 3 & 7 & 6 & 1 & 5 & 18 & $2 / 29=1 / 18+1 / 87_{3}+1 / 52218$ \\
\hline 5 & 11 & 8 & 3 & 5 & 24 & $2 / 37=1 / 24+1 / 111_{3}+1 / 296_{8}{ }^{E g}$ \\
\hline 3 & 7 & 6 & 1 & 5 & 24 & $2 / 41=1 / 24+1 / 164_{4}+1 / 98424$ \\
\hline 4 & 9 & 7 & 2 & 5 & 28 & $2 / 47=1 / 28+1 / 188_{4}+1 / 658_{14}$ \\
\hline 3 & 7 & 6 & 1 & 5 & 30 & $2 / 53=1 / 30+1 / 2655+1 / 159030$ \\
\hline 6 & 13 & 9 & 4 & 5 & 36 & $2 / 59=1 / 36+1 / 2364+1 / 5319^{E g}$ \\
\hline 3 & 7 & 6 & 1 & 5 & 48 & $2 / 89=1 / 48+1 / 712_{8}+1 / 427248$ \\
\hline 4 & 9 & 8 & 1 & 7 & 16 & $2 / 23=1 / 16+1 / 46_{2}+1 / 36816$ \\
\hline 6 & 13 & 10 & 3 & 7 & 30 & $2 / 47=1 / 30+1 / 141_{3}+1 / 470_{10}^{E g}$ \\
\hline 5 & 11 & 9 & 2 & 7 & 36 & $2 / 61=1 / 36+1 / 244_{4}+1 / 1098_{18}$ \\
\hline 4 & 9 & 8 & 1 & 7 & 40 & $2 / 71=1 / 40+1 / 355_{5}+1 / 284040$ \\
\hline 7 & 15 & 11 & 4 & 7 & 44 & $2 / 73=1 / 44+1 / 292_{4}+1 / 803_{11}$ \\
\hline 5 & 11 & 9 & 2 & 7 & 54 & $2 / 97=1 / 54+1 / 582_{6}+1 / 2619_{27}$ \\
\hline 5 & 11 & 10 & 1 & 9 & 20 & $2 / 29=1 / 20+1 / 58_{2}+1 / 580_{20}$ \\
\hline 6 & 13 & 11 & 2 & 9 & 22 & $2 / 31=1 / 22+1 / 62_{2}+1 / 341_{11}$ \\
\hline 5 & 11 & 10 & 1 & 9 & 50 & $2 / 89=1 / 50+1 / 445{ }_{5}+1 / 445050$ \\
\hline 7 & 15 & 13 & 2 & 11 & 26 & $2 / 37=1 / 26+1 / 74_{2}+1 / 481_{13}$ \\
\hline 6 & 13 & 12 & 1 & 11 & 36 & $2 / 59=1 / 36+1 / 177_{3}+1 / 2124_{36}$ \\
\hline 8 & 17 & 14 & 3 & 11 & 42 & $2 / 67=1 / 42+1 / 201_{3}+1 / 938_{14}$ \\
\hline 6 & 13 & 12 & 1 & 11 & 48 & $2 / 83=1 / 48+1 / 3324+1 / 398448$ \\
\hline 7 & 15 & 13 & 2 & 11 & 52 & $2 / 89=1 / 52+1 / 356_{4}+1 / 231426$ \\
\hline 7 & 15 & 14 & 1 & 13 & 28 & $2 / 41=1 / 28+1 / 82_{2}+1 / 1148_{28}$ \\
\hline 8 & 17 & 15 & 2 & 13 & 30 & $2 / 43=1 / 30+1 / 86_{2}+1 / 645_{15}$ \\
\hline 7 & 15 & 14 & 1 & 13 & 56 & $2 / 97=1 / 56+1 / 3884+1 / 543256$ \\
\hline 8 & 17 & 16 & 1 & 15 & 32 & $2 / 47=1 / 32+1 / 94_{2}+1 / 150432$ \\
\hline 8 & 17 & 16 & 1 & 15 & 48 & $2 / 79=1 / 48+1 / 237{ }_{3}+1 / 379248$ \\
\hline 9 & 19 & 18 & 1 & 17 & 36 & $2 / 53=1 / 36+1 / 106_{2}+1 / 190836$ \\
\hline 9 & 19 & 18 & 1 & 17 & 54 & $2 / 89=1 / 54+1 / 2673+1 / 4306_{54}$ \\
\hline 11 & 23 & 20 & 3 & 17 & 60 & $2 / 97=1 / 60+1 / 291_{3}+1 / 194020$ \\
\hline 10 & 21 & 20 & 1 & 19 & 40 & $2 / 59=1 / 40+1 / 118{ }_{2}+1 / 236040$ \\
\hline 11 & 23 & 21 & 2 & 19 & 42 & $2 / 61=1 / 42+1 / 122_{2}+1 / 1281_{21}$ \\
\hline 12 & 25 & 23 & 2 & 21 & 46 & $2 / 67=1 / 46+1 / 134_{2}+1 / 1541_{23}$ \\
\hline 12 & 25 & 24 & 1 & 23 & 48 & $2 / 71=1 / 48+1 / 142_{2}+1 / 340848$ \\
\hline 13 & 27 & 25 & 2 & 23 & 50 & $2 / 73=1 / 50+1 / 146_{2}+1 / 182525$ \\
\hline 14 & 29 & 27 & 2 & 25 & 54 & $2 / 79=1 / 54+1 / 158_{2}+1 / 2133_{27}$ \\
\hline 14 & 29 & 28 & 1 & 27 & 56 & $2 / 83=1 / 56+1 / 166_{2}+1 / 464856$ \\
\hline 15 & 31 & 30 & 1 & 29 & 60 & $2 / 89=1 / 60+1 / 178_{2}+1 / 534060$ \\
\hline 17 & 35 & 33 & 2 & 31 & 66 & $2 / 97=1 / 66+1 / 194_{2}+1 / 3201_{33}$ \\
\hline
\end{tabular}

As it is clear from Table 4 an obvious preference for the smallest $\Delta_{d}$ seems to be well followed.

After cut-off by $\mathrm{T}_{f}{ }^{[3]}=10$, Table 4 is reduced and allows us to analyze the following options:

Table 5. 3-terms options.

\begin{tabular}{|c|c|c|c|c|c|c|c|c|c|c|c|c|c|}
\hline \multicolumn{14}{|c|}{ Trials [3-terms] ordered with $\Delta_{\mathrm{d}} \nearrow$ showing where are the Egyptian options } \\
\hline & & & & & & & $\underline{\mathbf{n}}$ & $2 n+1$ & d2 & $\mathrm{d}_{3}$ & $\Delta_{d}$ & $D^{\mathrm{n}}{ }_{1}$ & [3-terms] decompositions $m_{3} \leq 10$ \\
\hline & & & & 1 & & & 1 & 3 & 2 & 1 & 1 & 8 & $2 / 13=1 / 8+1 / 524+1 / 1048^{E g}$ \\
\hline & & & & 1 & & & 1 & 3 & 2 & 1 & 1 & 10 & $2 / 17_{\mathrm{a}}=1 / 10+1 / 85_{5}+1 / 170_{10}$ \\
\hline & & & & 1 & & & 3 & 7 & 4 & 3 & 1 & 12 & $2 / 17_{b}=1 / 12+1 / 51_{3}+1 / 684^{E g} *$ \\
\hline & & & & 1 & & & 2 & 5 & 3 & 2 & 1 & 12 & $2 / 19=1 / 12+1 / 76{ }_{4}+1 / 114{ }_{6}^{E g}$ \\
\hline & & & & 1 & & & 2 & 5 & 3 & 2 & 1 & 18 & $2 / 31_{a}=1 / 18+1 / 186{ }_{6}+1 / 2799$ \\
\hline & & & & 1 & & & 4 & 9 & 5 & 4 & 1 & 20 & $2 / 31 b=1 / 20+1 / 124{ }_{4}+1 / 155{ }_{5}^{E g}$ \\
\hline & & & & & & & 3 & 7 & 4 & 3 & 1 & 24 & $2 / 41 \mathrm{~b}=1 / 24+1 / 2466+1 / 328{ }_{8}^{E g}$ \\
\hline & & & & & & & 4 & 9 & 5 & 4 & 1 & 40 & $2 / 71_{a}=1 / 40+1 / 568{ }_{8}+1 / 710{ }_{10} E g$ \\
\hline
\end{tabular}




\begin{tabular}{|c|c|c|c|c|c|c|}
\hline $\mathbf{n}$ & $2 n+1$ & $\mathbf{d}_{2}$ & d3 & $\Delta_{\mathrm{d}}$ & $D^{n}{ }_{1}$ & {$\left[3\right.$-terms] decompositions $m_{3} \leq 10$} \\
\hline 6 & 13 & 7 & 6 & 1 & 42 & $2 / 71 b=1 / 42+1 / 4266+1 / 4977$ \\
\hline 7 & 15 & 8 & 7 & 1 & 56 & $2 / 97 \mathrm{~b}=1 / 56+1 / 6797+1 / 7768^{E g} *$ \\
\hline 3 & 7 & 5 & 2 & 3 & 10 & $2 / 13=1 / 10+1 / 26_{2}+1 / 655$ \\
\hline 6 & 13 & 8 & 5 & 3 & 40 & $2 / 67 \mathrm{~b}=1 / 40+1 / 335{ }_{5}+1 / 5368_{8}^{E g}$ \\
\hline 4 & 9 & 7 & 2 & 5 & 14 & $2 / 19=1 / 14+1 / 38_{2}+1 / 133_{7}$ \\
\hline 5 & 11 & 8 & 3 & 5 & 24 & $2 / 37=1 / 24+1 / 111_{3}+1 / 296{ }_{8}^{E g}$ \\
\hline 6 & 13 & 9 & 4 & 5 & 36 & $2 / 59=1 / 36+1 / 2364+1 / 5319^{E g}$ \\
\hline 6 & 13 & 10 & 3 & 7 & 30 & $2 / 47=1 / 30+1 / 141_{3}+1 / 470_{10}{ }^{E g}$ \\
\hline
\end{tabular}

This table shows rare instances where multipliers $m_{2}, m_{3}$ are consecutive. It is always an interesting quality that does not require sophisticated mathematical justification. That will be denoted by a asterisk. Two instances are found also in [4-terms] series with $m_{2}, m_{3}, m_{4}$, see Sect. 6 .

Just as an indication, we display below the cases dropped out of a [3-terms] decomposition:

Table 6. Fractions to be broken down into 4-terms.

\begin{tabular}{lllllll}
\hline \multicolumn{6}{c}{ Table of trials [3-terms] for fractions to be broken down into 4-terms } \\
\hline $\mathbf{n}$ & $\mathbf{2 n + 1}$ & $\mathbf{d}_{\mathbf{2}}$ & $\mathbf{d}_{\mathbf{3}}$ & $\boldsymbol{\Delta}_{\mathbf{d}}$ & $\boldsymbol{D}_{\mathbf{1}}^{\mathbf{n}}$ & Possible [3-terms] decompositions \\
\hline 4 & 9 & 8 & 1 & 7 & 16 & $2 / 23=1 / 16+1 / 46_{2}+1 / 368_{16}$ \\
1 & 3 & 2 & 1 & 1 & 16 & $2 / 29=1 / 16+1 / 232_{8}+1 / 464_{16}$ \\
3 & 7 & 6 & 1 & 5 & 18 & $2 / 29=1 / 20+1 / 58_{2}+1 / 580_{20}$ \\
5 & 11 & 10 & 1 & 9 & 20 & $2 / 29=1 / 20+1 / 58_{2}+1 / 580_{20}$ \\
2 & 5 & 4 & 1 & 3 & 24 & $2 / 43=1 / 24+1 / 258_{6}+1 / 1032_{24}$ \\
2 & 5 & 3 & 2 & 1 & 24 & $2 / 43=1 / 24+1 / 344_{8}+1 / 516_{12}$ \\
8 & 17 & 15 & 2 & 13 & 30 & $2 / 43=1 / 30+1 / 86_{2}+1 / 645_{15}$ \\
1 & 3 & 2 & 1 & 1 & 32 & $2 / 61=1 / 32+1 / 976_{16}+1 / 1952$ \\
5 & 11 & 9 & 2 & 7 & 36 & $2 / 61=1 / 36+1 / 244_{4}+1 / 1098_{18}$ \\
11 & 23 & 21 & 2 & 19 & 42 & $2 / 61=1 / 42+1 / 122_{2}+1 / 1281_{21}$ \\
1 & 3 & 2 & 1 & 1 & 38 & $2 / 73=1 / 38+1 / 138_{19}+1 / 2274$ \\
3 & 7 & 5 & 2 & 3 & 40 & $2 / 73=1 / 40+1 / 154_{8}+1 / 1460_{20}$ \\
7 & 15 & 11 & 4 & 7 & 44 & $2 / 73=1 / 44+1 / 292_{4}+1 / 803_{11}$ \\
13 & 27 & 25 & 2 & 23 & 50 & $2 / 73=1 / 50+1 / 146_{2}+1 / 1825_{25}$ \\
\hline
\end{tabular}

Table of trials [3-terms] for fractions to be broken down into 4-terms

\begin{tabular}{lllllll}
\hline $\mathbf{n}$ & $\mathbf{2 n + 1}$ & $\mathbf{d}_{\mathbf{2}}$ & $\mathbf{d}_{\mathbf{3}}$ & $\boldsymbol{\Delta}_{\mathbf{d}}$ & $\boldsymbol{D}^{\mathbf{n}} \mathbf{1}$ & $\begin{array}{l}\text { Possible [3-terms] } \\
\text { decompositions }\end{array}$ \\
\hline 2 & 5 & 3 & 2 & 1 & 42 & $2 / 79=1 / 42+1 / 1106_{14}+1 / 1659_{21}$ \\
8 & 17 & 16 & 1 & 15 & 48 & $2 / 79=1 / 48+1 / 27_{3}+1 / 3792_{48}$ \\
14 & 29 & 27 & 2 & 25 & 54 & $2 / 79=1 / 54+1 / 158_{2}+1 / 2133_{27}$ \\
2 & 5 & 4 & 1 & 3 & 44 & $2 / 83=1 / 44+1 / 913_{11}+1 / 3652_{44}$ \\
6 & 13 & 12 & 1 & 11 & 48 & $2 / 83=1 / 48+1 / 32_{4}+1 / 3984_{48}$ \\
14 & 29 & 28 & 1 & 27 & 56 & $2 / 83=1 / 56+1 / 1_{2}+1 / 4646_{56}$ \\
1 & 3 & 2 & 1 & 1 & 46 & $2 / 89=1 / 46+1 / 2047_{23}+1 / 4094_{46}$ \\
3 & 7 & 6 & 1 & 5 & 48 & $2 / 89=1 / 48+1 / 712_{8}+1 / 4272_{48}$ \\
3 & 7 & 4 & 3 & 1 & 48 & $2 / 89=1 / 48+1 / 1068_{12}+1 / 1424_{16}$ \\
5 & 11 & 10 & 1 & 9 & 50 & $2 / 89=1 / 50+1 / 445_{5}+1 / 4450$ \\
7 & 15 & 13 & 2 & 11 & 52 & $2 / 89=1 / 52+1 / 356_{4}+1 / 2314_{26}$ \\
9 & 19 & 18 & 1 & 17 & 54 & $2 / 89=1 / 54+1 / 267_{3}+1 / 4306_{54}$ \\
15 & 31 & 30 & 1 & 29 & 60 & $2 / 89=1 / 60+1 / 178_{2}+1 / 5340_{60}$ \\
\hline
\end{tabular}

Our definition of $\mathrm{T}_{f}$ does not depend on a arbitrary value of $D_{3}$ fixed to 1000 as often assumed in the literature. It depends only on the circumstances imposed by the current project. Subdivide now Table 5 into 3 sets according to the properties of each $D$. A first with a only one $\Delta_{d}$, a second with two different $\Delta_{d}$ and a third with two conflicting identical $\Delta_{d}$. That yields:
Table 7. A single $\Delta_{d}$ [3-terms].

\begin{tabular}{lllllll}
\hline \multicolumn{3}{l}{$\boldsymbol{D}$ with a single } & $\boldsymbol{\Delta}_{\mathbf{d}}$ (options: $\mathbf{n}$ ) & \multicolumn{2}{c}{ Scribes's decision: obvious } \\
\hline $\mathbf{n}$ & $\mathbf{2 n + 1}$ & $\mathbf{d}_{\mathbf{2}}$ & $\mathbf{d}_{\mathbf{3}}$ & $\boldsymbol{\Delta}_{\mathbf{d}}$ & $\boldsymbol{D}_{\mathbf{1}}^{\mathbf{n}}$ & [3-terms] decomposition \\
\hline 3 & 7 & 4 & 3 & 1 & 24 & $2 / 41=1 / 24+1 / 246_{6}+1 / 328_{8} \mathrm{Eg}$ \\
7 & 15 & 8 & 7 & 1 & 56 & $2 / 97=1 / 56+1 / 679_{7}+1 / 776_{8} \mathrm{Eg} *$ \\
6 & 13 & 8 & 5 & 3 & 40 & $2 / 67=1 / 40+1 / 335_{5}+1 / 536_{8} \mathrm{Eg}$ \\
5 & 11 & 8 & 3 & 5 & 24 & $2 / 37=1 / 24+1 / 111_{3}+1 / 296_{8} \mathrm{Eg}$ \\
6 & 13 & 9 & 4 & 5 & 36 & $2 / 59=1 / 36+1 / 236_{4}+1 / 531_{9} \mathrm{Eg}$ \\
6 & 13 & 10 & 3 & 7 & 30 & $2 / 47=1 / 30+1 / 141_{3}+1 / 470_{10} \mathrm{Eg}$ \\
\hline
\end{tabular}

Table 8. Two different $\Delta_{d}$ [3-terms].

\begin{tabular}{lllllll}
\hline \multicolumn{1}{l}{$\boldsymbol{D}$ with two different $\boldsymbol{\Delta}_{\mathbf{d}}$ (options: yes) } \\
\hline \multicolumn{10}{c}{ Scribes's decision: smallest $\boldsymbol{\Delta}_{\mathbf{d}}$} \\
\hline $\mathrm{n}$ & $2 \mathrm{n}+1$ & $\mathrm{~d}_{2}$ & $\mathrm{~d}_{3}$ & $\Delta_{\mathrm{d}}$ & $D^{\mathrm{n}}{ }_{1}$ & {$[$ 3-terms]decompositions } \\
1 & 3 & 2 & 1 & 1 & 8 & $2 / 13=1 / 8+1 / 52_{4}+1 / 104_{8}{ }^{E g}$ \\
3 & 7 & 5 & 2 & 3 & 10 & $2 / 13=1 / 10+1 / 26_{2}+1 / 65_{5}$ \\
2 & 5 & 3 & 2 & 1 & 12 & $2 / 19=1 / 12+1 / 76_{4}+1 / 114_{6}{ }^{E g}$ \\
4 & 9 & 7 & 2 & 5 & 14 & $2 / 19=1 / 14+1 / 38_{2}+1 / 133_{7}$ \\
\hline
\end{tabular}

Table 9. Two conflicting identical $\Delta_{d}$ [3-terms].

\begin{tabular}{|c|c|c|c|c|c|c|}
\hline \multicolumn{7}{|c|}{$D$ with two conflicting identical $\Delta_{\mathrm{d}}$ (options: yes) } \\
\hline \multirow[b]{2}{*}{$\mathrm{n}$} & \multirow[b]{2}{*}{$2 n+1$} & \multirow[b]{2}{*}{$\mathrm{d}_{2}$} & \multirow[b]{2}{*}{$\mathrm{d}_{3}$} & \multirow[b]{2}{*}{$\Delta_{\mathrm{d}}$} & \multirow[b]{2}{*}{$D^{\mathrm{n}}{ }_{1}$} & $\begin{array}{l}\text { Scribes's decision: consecutive } \\
\text { multipliers }\end{array}$ \\
\hline & & & & & & [3-terms] decompositions \\
\hline 1 & 3 & 2 & 1 & 1 & 10 & $2 / 17_{a}=1 / 10+1 / 85_{5}+1 / 170_{10}$ \\
\hline 3 & 7 & 4 & 3 & 1 & 12 & $2 / 17_{\mathrm{b}}=1 / 12+1 / 51_{3}+1 / 68_{4} E g *$ \\
\hline 2 & 5 & 3 & 2 & 1 & 18 & $2 / 31_{a}=1 / 18+1 / 186_{6}+1 / 2799$ \\
\hline \multirow[t]{2}{*}{4} & 9 & 5 & 4 & 1 & 20 & $2 / 31_{\mathrm{b}}=1 / 20+1 / 124_{4}+1 / 1555^{E g} *$ \\
\hline & & & & & & Scribes's decision: $2 \mathrm{n} \leq 10$ \\
\hline n & $2 n+1$ & $\mathbf{d}_{\mathbf{2}}$ & $\mathbf{d}_{3}$ & $\Delta_{d}$ & $D^{n}{ }_{1}$ & [3-terms] decompositions \\
\hline 4 & 9 & 5 & 4 & 1 & 40 & $2 / 71_{a}=1 / 40+1 / 568_{8}+1 / 710_{10}$ \\
\hline 6 & 13 & 7 & 6 & 1 & 42 & $2 / 71_{b}=1 / 42+1 / 426_{6}+1 / 4977_{7} *$ \\
\hline
\end{tabular}

Remark: in the cases involving options possible, and in these cases only, the solutions for

$\{2 / \mathrm{D}=2 / 13,2 / 19,2 / 17,2 / 31\}$ were chosen respectively in the set $\{n=1,2,3,4\}_{\mid 2 n \leq 10}$.

For ruling on $2 / 71$ there is no convincing arithmetical argumentation, then the choice could have been the simplicity and direct observation: once again a boundary like $2 n \leq 10$ is used for picking $n=4$. That's it.

Too simple, but what else could be simpler?

After this natural selection by cut-off with a Top-flag $\mathrm{T}_{\mathrm{f}}{ }^{[3]}=$ 10 and appropriate decisions, it remains some cases to be examined, especially these with $10<m_{3} \leq 16$

because of the singular status of $2 / 23$, that the scribes will retain with a decomposition into 2 terms. We display below these cases. Of course 2/61, 2/83 are ex officio excluded from the analysis. 
(Anticipation is made on [4-terms] analysis, see Sect. 6 follow, like $\mathrm{T}_{f}^{[4]}=10$ ). below for definition of $\Delta^{\prime}{ }_{d}=d_{3}-d_{4}$ and related decisions that

Table 10. Dynamic comparison for transitions $3=>4$.

Unique [2-terms] solution $2 / 23=1 / 12+1 / 276_{12}{ }^{E g}$

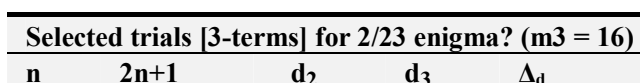

\begin{tabular}{lllllll}
\hline $\mathbf{n}$ & $\mathbf{2 n + 1}$ & $\mathbf{d}_{\mathbf{2}}$ & $\mathbf{d}_{\mathbf{3}}$ & $\boldsymbol{\Delta}_{\mathbf{d}}$ & $\boldsymbol{D}_{\mathbf{1}_{1}}$ & Unique [3-terms] decomposition \\
\hline 4 & 9 & 8 & 1 & 7 & 16 & $2 / 23=1 / 16+1 / 46_{2}+1 / 368_{16}$ \\
\hline
\end{tabular}

\begin{tabular}{llllllll}
\hline \multicolumn{2}{l}{ Selected trials [4-terms] } & $\mathbf{2} / \mathbf{2 3}$ & & & & \\
\hline $\mathbf{n}$ & $\mathbf{2 n}+\mathbf{1}$ & $\mathbf{d}_{\mathbf{2}}$ & $\mathbf{d}_{\mathbf{3}}$ & $\mathbf{d}_{\mathbf{4}}$ & $\boldsymbol{\Delta}_{\mathbf{d}}$ & $\mathbf{D}^{\mathbf{n}_{1}}$ & [4-terms] decomposition $\mathbf{m} \mathbf{4} \leq \mathbf{1 0}$ \\
\hline 8 & 17 & 10 & 5 & 2 & 3 & 20 & $2 / 23=1 / 20+1 / 46_{2}+1 / 92_{4}+1 / 230_{10}$ \\
\hline
\end{tabular}

\begin{tabular}{|c|c|c|c|c|c|c|}
\hline \multicolumn{7}{|c|}{ Selected trials [3-terms] 2/29 $\left(\mathrm{m}_{3}=16\right)$} \\
\hline $\mathbf{n}$ & $2 n+1$ & $d_{2}$ & $d_{3}$ & $\Delta_{\mathrm{d}}$ & $D_{1}^{\mathrm{n}}$ & Possible [3-terms] decomposition \\
\hline 1 & 3 & 2 & 1 & 1 & 16 & $2 / 29=1 / 16+1 / 232_{8}+1 / 46416$ \\
\hline
\end{tabular}

\begin{tabular}{|c|c|c|c|c|c|c|c|}
\hline \multicolumn{8}{|c|}{ Selected trials [4-terms] $2 / 29$} \\
\hline n & $2 n+1$ & $d_{2}$ & $d_{3}$ & $\mathbf{d}_{4}$ & $\Delta_{\mathbf{d}_{\mathrm{d}}}^{\prime}$ & $D^{\mathrm{n}}{ }_{1}$ & Possible [4-terms] decompositions $\mathrm{m} 4 \leq 10$ \\
\hline 9 & 19 & 12 & 4 & 3 & 1 & 24 & $2 / 29=1 / 24+1 / 58_{2}+1 / 174_{6}+\underline{1 / 232}{ }_{8}^{E g}$ \\
\hline 5 & 11 & 5 & 4 & 2 & 2 & 20 & $2 / 29=1 / 20+1 / 116_{4}+1 / 145_{5}+1 / 290_{10}$ \\
\hline 15 & 31 & 15 & 10 & 6 & 4 & 30 & $2 / 29=1 / 30+1 / 58_{2}+1 / 87_{3}+1 / 145_{5}$ \\
\hline
\end{tabular}

\begin{tabular}{|c|c|c|c|c|c|c|}
\hline \multicolumn{7}{|c|}{ Selected trials [3-terms] $2 / 89\left(\mathrm{~m}_{3}=16\right)$} \\
\hline $\mathbf{n}$ & $2 n+1$ & $\mathbf{d}_{2}$ & $d_{3}$ & $\Delta_{\mathrm{d}}$ & $D^{\mathrm{n}}{ }_{1}$ & Possible [3-terms] decomposition $\left(\mathrm{m}_{3}=16\right)$ \\
\hline 3 & 7 & 4 & 3 & 1 & 48 & $2 / 89 b=1 / 48+1 / 106812+1 / 142416$ \\
\hline
\end{tabular}

\begin{tabular}{llllllll}
\hline \multicolumn{2}{l}{ Selected trials [4-terms] } & $\mathbf{2 / 8 9}$ \\
$\mathbf{n}$ & $\mathbf{2 n + 1}$ & $\mathbf{d}_{\mathbf{2}}$ & $\mathbf{d}_{\mathbf{3}}$ & $\mathbf{d}_{\mathbf{4}}$ & $\boldsymbol{\Delta}_{\mathbf{d}}$ & $\boldsymbol{D}^{\mathbf{n}}{ }_{1}$ & Possible [4-terms] decompositions $\mathbf{m}_{\mathbf{4}} \leq \mathbf{1 0}$ \\
\hline 15 & 31 & 15 & 10 & 6 & 4 & 60 & $2 / 89=1 / 60+1 / 356_{4}+1 / 534_{6}+1 / 890_{10}{ }^{E g}$ \\
\hline
\end{tabular}

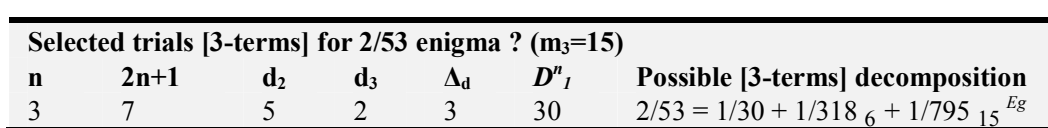

\begin{tabular}{|c|c|c|c|c|c|c|}
\hline \multicolumn{7}{|c|}{ Selected trials [4-terms] for $2 / 53$} \\
\hline$\underline{\mathbf{n}}$ & $2 n+1$ & $\mathbf{d}_{2}$ & $\mathbf{d}_{3}$ & $\mathbf{d}_{4}$ & $\Delta \Delta_{\mathrm{d}}^{\prime}$ & [4-terms] decomposition $m_{4} \leq 10$ \\
\hline 9 & 19 & 9 & 6 & 4 & 2 & $2 / 53=1 / 36+1 / 212_{4}+1 / 318_{6}+1 / 4779$ \\
\hline \multicolumn{7}{|c|}{ Selected trials [3-terms] $2 / 43\left(\left(m_{3}=12\right)\right.$ or $\left(\left(m_{3}=15\right)\right.$} \\
\hline $\mathbf{n}$ & $2 n+1$ & $\mathbf{d}_{2}$ & $\mathbf{d}_{3}$ & $\Delta_{\mathrm{d}}$ & $D_{1}^{\mathrm{n}}$ & Possible [3-terms] decompositions \\
\hline 2 & 5 & 3 & 2 & 1 & 24 & $2 / 43=1 / 24+1 / 344_{8}+1 / 516_{12}$ \\
\hline 8 & 17 & 15 & 2 & 13 & 30 & $z / 43=1 / 30+1 / 86_{z}+1 / 645_{15}$ \\
\hline
\end{tabular}

\begin{tabular}{|c|c|c|c|c|c|c|c|}
\hline \multicolumn{8}{|c|}{ Selected trials [4-terms] $2 / 43$} \\
\hline $\mathbf{n}$ & $2 n+1$ & $\mathbf{d}_{2}$ & $\mathbf{d}_{3}$ & $\mathbf{d}_{4}$ & $\Delta_{\mathbf{d}^{\prime}}$ & $D^{n_{1}}$ & [4-terms] decomposition $\mathrm{m}_{4} \leq 10$ \\
\hline 20 & 41 & 21 & 14 & 6 & 8 & 42 & $2 / 43=1 / 42+1 / 86_{2}+1 / 129_{3}+1 / 3017^{E g}$ \\
\hline
\end{tabular}

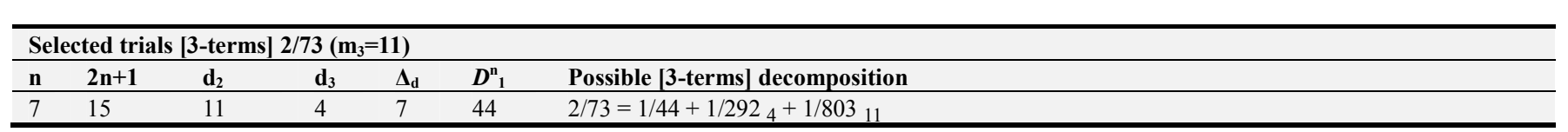

\begin{tabular}{llllllll}
\hline \multicolumn{7}{l}{ Selected trials [4-terms] } & $\mathbf{2} / \mathbf{7 3}$ \\
$\mathbf{n}$ & $\mathbf{2 n + 1}$ & $\mathbf{d}_{\mathbf{2}}$ & $\mathbf{d}_{\mathbf{3}}$ & $\mathbf{d}_{\mathbf{4}}$ & $\boldsymbol{\Delta}_{\mathbf{}_{\mathbf{d}}}$ & $\boldsymbol{D}^{\mathbf{n}}{ }_{\mathbf{1}}$ & {$\left[\right.$ [-terms] decomposition $\mathbf{m}_{\mathbf{4}} \leq \mathbf{1 0}$} \\
\hline 23 & 47 & 20 & 15 & 12 & 3 & 60 & $2 / 73_{\mathrm{c}}=1 / 60+1 / 219_{3}+1 / 292_{4}+1 / 365_{5}{ }^{\mathrm{Eg}} *$ \\
\hline
\end{tabular}

We repeat that we are always in a logic of a construction site with difficulties arising in different parts of the project. Problems are processed case after case and do not interfere with another previous part. If not, all becomes incomprehensible. A overview supervised by a chief scribe can not be conflicted. The 6 cases presented above confront us with a dynamic alternative: select the transition from 3 to 4 fractions, or reject it. This exceptional situation is new in the 
table construction project, as well as the solution itself!

It can be observed that 5 cases on 6 have in common the fact that a same denominator appears in [3-terms] and [4-terms] decompositions.

A priori, this fact may be seen as not being an improvement to better decompose a [3-terms]. Unless we find a real improvement worthwhile.

2/89: sixth case, out of the category 'same denominator', is quickly ruled and [4-terms]

decomposition is adopted. (Anyway it belonged to this table only because $\left.m_{3}=16\right)$.

2/43: once dropped out the option $m_{3}=15$, due to a too high gap $\Delta_{d}=13$, the same argument holds, then [4-terms] decomposition is adopted.

2/73: the [4-terms] expansion provides an improvement since that leads to three consecutive multipliers $\{3,4,5\}$, thus this solution is adopted.

Three cases (slightly reordered) remain to be solved, they are displayed just below.

Table 11. Cases 2/23, 2/53 and 2/29

\section{Unique [2-terms] solution \\ $2 / 23=1 / 12+1 / 276_{12}{ }^{E g}$}

\begin{tabular}{|c|c|c|c|c|c|c|}
\hline \multicolumn{7}{|c|}{ Selected trials $\left[3\right.$-terms] for $2 / 23$ enigma? $\left(m_{3}=16\right)$} \\
\hline $\mathbf{n}$ & $2 n+1$ & $d_{2}$ & $d_{3}$ & $\Delta_{\mathrm{d}}$ & $D^{\mathrm{n}}{ }_{1}$ & Unique [3-terms] decomposition \\
\hline 4 & 9 & 8 & 1 & 7 & 16 & $2 / 23=1 / 16+1 / \underline{46}_{2}+1 / 36816$ \\
\hline \multicolumn{7}{|c|}{ Selected trials $[4-t e r m s] 2 / 23$} \\
\hline $\mathbf{n}$ & $2 n+1$ & $\mathbf{d}_{2}$ & $\mathbf{d}_{3}$ & $\mathbf{d}_{4}$ & $\Delta_{\mathbf{d}^{\prime}}$ & [4-terms] decomposition $m_{4} \leq \mathbf{1 0}$ \\
\hline 8 & 17 & 10 & 5 & 2 & 3 & $2 / 23=1 / 20+1 / 46_{2}+1 / 92_{4}+1 / 230_{10}$ \\
\hline
\end{tabular}

\begin{tabular}{lllllll}
\hline \multicolumn{6}{l}{ Selected trials } & [3-terms] for $\mathbf{2 / 5 3}$ enigma? $\left(\mathbf{m}_{\mathbf{3}}=\mathbf{1 5}\right)$ \\
\hline $\mathbf{n}$ & $\mathbf{2 n}+\mathbf{1}$ & $\mathbf{d}_{\mathbf{2}}$ & $\mathbf{d}_{\mathbf{3}}$ & $\boldsymbol{\Delta}_{\mathbf{d}}$ & $\boldsymbol{D}^{\mathbf{n}} \mathbf{1}_{1}$ & Possible [3-terms] decomposition \\
\hline 3 & 7 & 5 & 2 & 3 & 30 & $2 / 53=1 / 30+1 / \underline{118}_{6}+1 / 795_{15}{ }^{\mathrm{Eg}}$ \\
\hline
\end{tabular}

\begin{tabular}{|c|c|c|c|c|c|c|c|}
\hline \multicolumn{8}{|c|}{ Selected trials [4-terms] for $2 / 53$} \\
\hline $\mathrm{n}$ & $2 n+1$ & $d_{2}$ & $d_{3}$ & $d_{4}$ & $\Delta_{\mathrm{d}}^{\prime}$ & $D^{\mathrm{n}}{ }_{1}$ & [4-terms] decomposition $m_{4} \leq 10$ \\
\hline 9 & 19 & 9 & 6 & 4 & 2 & 36 & $2 / 53=1 / 36+1 / 212_{4}+1 / 3186+1 / 477 q$ \\
\hline
\end{tabular}

\begin{tabular}{|c|c|c|c|c|c|c|}
\hline \multicolumn{7}{|c|}{ Selected trials [3-terms] $2 / 29\left(m_{3}=16\right)$} \\
\hline$n$ & $2 n+1$ & $d_{2}$ & $d_{3}$ & $\Delta_{\mathrm{d}}$ & $\mathbf{D}^{\mathrm{n}}$ & Possible [3-terms] decomposition \\
\hline 1 & 3 & 2 & 1 & 1 & 16 & $2 / 29=1 / 16+1 / 232_{8}+1 / 46416$ \\
\hline \multicolumn{7}{|c|}{ Selected trials [4-terms] 2/29 } \\
\hline $\mathrm{n}$ & $2 n+1$ & $\mathbf{d}_{2}$ & $\mathbf{d}_{3}$ & d & $\Delta_{\mathbf{d}^{\prime}}$ & Possible [4-terms] decompositions $m_{4} \leq 10$ \\
\hline 9 & 19 & 12 & 4 & 3 & 1 & $2 / 29_{a}=1 / 24+1 / 58_{2}+1 / 174{ }_{6}+1 / 2328^{E g}$ \\
\hline 5 & 11 & 5 & 4 & 2 & 2 & $2 / 29 \mathrm{~b}=1 / 20+1 / 116_{4}+1 / 145_{5}+1 / 290_{10}$ \\
\hline 15 & 31 & 15 & 10 & 6 & 4 & $2 / 29_{c}=1 / 30+1 / 58_{2}+1 / 87_{3}+1 / 145_{5}$ \\
\hline
\end{tabular}

For each fraction identical denominators (underlined inside a box) have a well defined position in a [3-terms] expansion and another in a [4-terms]. We denote respectively these positions by rank [3] and rank [4]. Same denominators will be denoted by 'same $D_{\mathrm{i}}$ '.

The schema below summarizes the situation.

Table 12. Ranks positions.

\begin{tabular}{lllll}
\hline Fraction & same $_{\mathbf{i}}$ & rank $^{[3]}$ & rank $^{[4]}$ & Appreciation on ranks \\
\hline $2 / 23$ & 46 & 2 & 2 & no interest \\
\hline $2 / 53$ & 318 & 2 & 3 & too near \\
$2 / 29_{a}$ & 232 & 2 & 4 & acceptable + smallest $\Delta_{d}^{\prime}$ \\
\hline
\end{tabular}

Some convenient rulings ensue, namely

$2 / 23$; no solution; then come back to the only one solution in 2 terms.

2/53; maintain [3-terms] solution; reject [4-terms] solution. 2/29; adopt [4-terms] solution.

\section{6. [4-Terms] Analysis}

Right now consider the [4-terms] cases. Egyptians gave:

Table 13. [4-terms] with $2 D_{1}$ decomposition

\begin{tabular}{lll}
\hline Ahmes's selections [4-terms] & $\Leftarrow$ & Unity decomposition \\
\hline $2 / 29=1 / 24+1 / 58_{2}+1 / 174_{6}+1 / 232_{8}$ & $\Leftarrow$ & $48=29+12+4+3$ \\
$2 / 43=1 / 42+1 / 86_{2}+1 / 129_{3}+1 / 301_{7}$ & $\Leftarrow$ & $84=43+21+14+6$ \\
$2 / 61=1 / 40+1 / 244_{4}+1 / 488_{8}+1 / 610_{10}$ & $\Leftarrow$ & $80=61+10+5+4$ \\
$2 / 73=1 / 60+1 / 219_{3}+1 / 292_{4}+1 / 365_{5}$ & $\Leftarrow$ & $120=73+20+15+12$ \\
$2 / 79=1 / 60+1 / 237_{3}+1 / 36_{4}+1 / 790_{10}$ & $\Leftarrow$ & $120=79+20+15+6$ \\
\hline
\end{tabular}




\begin{tabular}{lll}
\hline Ahmes's selections [4-terms] & $\Leftarrow$ & Unity decomposition \\
\hline $2 / 83=1 / 60+1 / 332_{4}+1 / 415_{5}+1 / 498_{6}$ & $\Leftarrow$ & $120=83+15+12+10$ \\
$2 / 89=1 / 60+1 / 356_{4}+1 / 534_{6}+1 / 890_{10}$ & $\Leftarrow$ & $120=89+15+10+6$ \\
\hline
\end{tabular}

The task of finding $D_{l}$ is rather simple, from the moment when one realizes that it is enough to establish a table of odd numbers $(2 n+1)_{\mid n \geq 3}$ as a sum of three numbers $d_{2}+d_{3}+d_{4}$ with $d_{2}>d_{3}>d_{4}$. This is easy to do and independent of any context. The table contains exactly $([\mathrm{n} / 2][(\mathrm{n}+1) / 2]-1)$ triplets $\left\{d_{1}, d_{2}, d_{3}\right\}$ and $\sup \left(d_{2}\right)=2 \mathrm{n}-2$. Square brackets here [ ] means 'integer part of'. One can start with the lowest values as follows: $d_{4}=1, d_{3}=2,3,4, \cdots, d_{2}=3,4,5, \cdots ; d_{4}=2, d_{3}=3,4,5, \cdots$, $d_{2}=4,5,6, \cdots$ and so on, with the condition $d_{3}+d_{2}=d_{4}+1 \bmod (2)$.

From (4) the first candidate possible for $D_{l}$ starts at the value $D_{l}{ }^{0}=(D+1) 2$. As for Sect. 5 we can search for general solutions of the form

$$
D_{l}^{n}=D_{1}^{0}+n,
$$

whence

$$
2 D_{1}{ }^{n}-D=2 n+1=d_{2}+d_{3}+d_{4} .
$$

From the first table of triplets, a new table (of trials) is built, where this time triplets are selected if

$d_{1}, d_{2}, d_{3}$ divide $\left(D+d_{2}+d_{3}+d_{4}\right) / 2$. This provides a $D_{1}{ }^{n}$ possible. In this favorable case, first $D_{4}$ is calculated by $D D_{1} / d_{4}$, then $D_{3}$ by $D D_{I} / d_{3}$, and $D_{2}$ by $D D_{1} / d_{2}$.

This table of trials, properly defined by the equation just below (included the constraints), ie

$$
2 n+1=d_{2}+d_{3}+d_{4} .
$$

This is obviously a bit longer to establish than for doublets. By simplicity $D_{l}{ }^{n}$ will be not written as
$D_{l}^{n}\left(d_{2}, d_{3}, d_{4}\right)$. For decompositions into 4 terms the total of trials yields only 71 possibilities !

Of course our remark previously made about doublets is still valid for triplets. Likewise, Abdulaziz's parameter $[R]$ takes the form

$$
\left.[R]=1 /\left[\left(D_{l} / d_{2}\right)\right]+1 /\left[\left(D_{l} / d_{3}\right)\right)\right]+1 /\left[\left(D_{l} / d_{4}\right)\right] .
$$

The notation used in our tables will be

$$
\Delta_{d}^{\prime}=d_{3}-d_{4}
$$

Chief scribe wisely decided to impose a upper bound to all the denominators $D_{4}$, such that

$$
D_{4} \leq D \mathbf{T}_{f}^{[4]} \text {. }
$$

This cut-off beyond $\mathbf{T}_{f}{ }^{[4]}$ is equivalent to a mathematical condition on $D_{l}$ :

$$
D_{1} \leq d_{4} \mathbf{T}_{f}{ }^{[4]} .
$$

Here again, choosing $\mathrm{T}_{f}\left[{ }^{[4]}=10\right.$ is quite appropriate. Thus a general coherence is ensured throughout the project, since 11 out of 12 decompositions into 3 terms were solved with $\mathrm{T}_{f}^{[3]}=$ 10 .

\begin{tabular}{|c|c|c|c|c|c|c|c|}
\hline $\mathbf{n}$ & $2 n+1$ & $\mathbf{d}_{2}$ & $\mathbf{d}_{3}$ & $\mathbf{d}_{4}$ & $\Delta_{d}^{\prime}$ & $D_{1}^{\mathrm{n}}$ & Possible [4-terms] decompositions \\
\hline 9 & 19 & 12 & 4 & 3 & 1 & 24 & $2 / 29=1 / 24+1 / 58_{2}+1 / 174_{6}+1 / 232_{8}{ }^{E g}$ \\
\hline 5 & 11 & 6 & 3 & 2 & 1 & 36 & $2 / 61_{a}=1 / 36+1 / 366_{6}+1 / 73212+1 / 1098_{18}$ \\
\hline 9 & 19 & 10 & 5 & 4 & 1 & 40 & $2 / 61_{\mathrm{b}}=1 / 40+1 / 244_{4}+1 / 488_{8}+1 / 610_{10} E g$ \\
\hline 5 & 11 & 6 & 3 & 2 & 1 & 42 & $2 / 73_{b}=1 / 42+1 / 511_{7}+1 / 1022_{14}+1 / 1533_{21}$ \\
\hline 11 & 23 & 16 & 4 & 3 & 1 & 48 & $2 / 73_{c}=1 / 48+1 / 219_{3}+1 / 876_{12}+1 / 1168_{16}$ \\
\hline 8 & 17 & 12 & 3 & 2 & 1 & 48 & $2 / 79_{a}=1 / 48+1 / 316_{4}+1 / 1264_{16}+1 / 1896_{24}$ \\
\hline 6 & 13 & 8 & 3 & 2 & 1 & 48 & $2 / 83_{a}=1 / 48+1 / 498_{6}+1 / 132816+1 / 199224$ \\
\hline 6 & 13 & 6 & 4 & 3 & 1 & 48 & $2 / 83_{b}=1 / 48+1 / 664_{8}+1 / 996_{12}+1 / 1328_{16}$ \\
\hline 14 & 29 & 14 & 8 & 7 & 1 & 56 & $2 / 83_{c}=1 / 56+1 / 332_{4}+1 / 581_{7}+1 / 664_{8}$ \\
\hline 18 & 37 & 30 & 4 & 3 & 1 & 60 & $2 / 83_{d}=1 / 60+1 / 166_{2}+1 / 1245_{15}+1 / 1660_{20}$ \\
\hline 3 & 7 & 4 & 2 & 1 & 1 & 48 & $2 / 89_{a}=1 / 48+1 / 1068_{12}+1 / 2136_{24}+1 / 427248$ \\
\hline 15 & 31 & 20 & 6 & 5 & 1 & 60 & $2 / 89_{b}=1 / 60+1 / 267_{3}+1 / 890_{5}+1 / 1068_{12}$ \\
\hline 6 & 13 & 9 & 3 & 1 & 2 & 18 & $2 / 23=1 / 18+1 / 46_{2}+1 / 138_{6}+1 / 414_{18}$ \\
\hline 5 & 11 & 5 & 4 & 2 & 2 & 20 & $2 / 29=1 / 20+1 / 116_{4}+1 / 145_{5}+1 / 290_{10}$ \\
\hline 8 & 17 & 13 & 3 & 1 & 2 & 39 & $2 / 61=1 / 39+1 / 183_{3}+1 / 793_{13}+1 / 2379_{39}$ \\
\hline 5 & 11 & 7 & 3 & 1 & 2 & 42 & $2 / 73_{a}=1 / 42+1 / 438_{6}+1 / 1022_{14}+1 / 3066_{42}$ \\
\hline 8 & 17 & 9 & 5 & 3 & 2 & 45 & $2 / 73_{b}=1 / 45+1 / 3655_{5}+1 / 6579+1 / 109515$ \\
\hline 18 & 37 & 15 & 12 & 10 & 2 & 60 & $2 / 83=1 / 60+1 / 3324+1 / 415{ }_{5}+1 / 4986^{E g}$ \\
\hline 18 & 37 & 21 & 9 & 7 & 2 & 63 & $2 / 89=1 / 63+1 / 267_{3}+1 / 623_{7}+1 / 801_{9}$ \\
\hline 8 & 17 & 10 & 5 & 2 & 3 & 20 & $2 / 23=1 / 20+1 / 46_{2}+1 / 92_{4}+1 / 230_{10}$ \\
\hline 8 & 17 & 10 & 5 & 2 & 3 & 30 & $2 / 43_{a}=1 / 30+1 / 129_{3}+1 / 258_{6}+1 / 645_{15}$ \\
\hline 10 & 21 & 16 & 4 & 1 & 3 & 32 & $2 / 43_{b}=1 / 32+1 / 86_{2}+1 / 344_{8}+1 / 1376_{32}$ \\
\hline 5 & 11 & 6 & 4 & 1 & 3 & 36 & $2 / 61_{\mathrm{a}}=1 / 36+1 / 366_{6}+1 / 549_{9}+1 / 2196_{36}$ \\
\hline
\end{tabular}

Remark that the condition (5.7) might be exploited from the beginning of the calculations for avoiding to handle too large denominators $D_{4}$. Simply find $d_{4}$, find $d_{3}$, find $d_{2}$, calculate $D_{l}$, if (5.7) is not fulfilled then quit, do not calculate $D_{4}, D_{3}, D_{2}$ and go to next values for $d_{4}, d_{3}, d_{2}, D_{1}$ and so on.

Table 14. Table of trials [4-terms] with increasing order of $\triangle{ }^{\prime}{ }_{d}$ only 71 possibilities. 


\begin{tabular}{|c|c|c|c|c|c|c|c|}
\hline $\mathbf{n}$ & $2 n+1$ & $\mathbf{d}_{2}$ & $\mathbf{d}_{3}$ & $d_{4}$ & $\Delta_{\mathrm{d}}^{\prime}$ & $D_{1}^{n_{1}}$ & Possible [4-terms] decompositions \\
\hline 11 & 23 & 14 & 6 & 3 & 3 & 42 & $2 / 61_{b}=1 / 42+1 / 183_{3}+1 / 427_{7}+1 / 854_{14}$ \\
\hline 13 & 27 & 22 & 4 & 1 & 3 & 44 & $2 / 61_{c}=1 / 44+1 / 1222+1 / 671_{11}+1 / 268444$ \\
\hline 15 & 31 & 26 & 4 & 1 & 3 & 52 & $2 / 73_{a}=1 / 52+1 / 146_{2}+1 / 94913+1 / 379652$ \\
\hline 19 & 39 & 28 & 7 & 4 & 3 & 56 & $2 / 73_{b}=1 / 56+1 / 146_{2}+1 / 5848+1 / 102214$ \\
\hline 23 & 47 & 20 & 15 & 12 & 3 & 60 & $2 / 73_{\mathrm{c}}=1 / 60+1 / 219{ }_{3}+1 / 292_{4}+1 / 365_{5} \mathrm{Eg}$ \\
\hline 8 & 17 & 12 & 4 & 1 & 3 & 48 & $2 / 79_{a}=1 / 48+1 / 3164+1 / 94812+1 / 379248$ \\
\hline 8 & 17 & 8 & 6 & 3 & 3 & 48 & $2 / 79_{b}=1 / 48+1 / 474_{6}+1 / 632_{8}+1 / 1264_{16}$ \\
\hline 16 & 33 & 28 & 4 & 1 & 3 & 56 & $2 / 79_{c}=1 / 56+1 / 158_{2}+1 / 1106_{14}+1 / 442456$ \\
\hline 6 & 13 & 8 & 4 & 1 & 3 & 48 & $2 / 83_{\mathrm{a}}=1 / 48+1 / 498_{6}+1 / 996_{12}+1 / 3984_{48}$ \\
\hline 8 & 17 & 10 & 5 & 2 & 3 & 50 & $2 / 83_{b}=1 / 50+1 / 415_{5}+1 / 830_{10}+1 / 2075_{25}$ \\
\hline 18 & 37 & 30 & 5 & 2 & 3 & 60 & $2 / 83_{c}=1 / 60+1 / 166_{2}+1 / 99612+1 / 249030$ \\
\hline 15 & 31 & 15 & 10 & 6 & 4 & 30 & $2 / 29=1 / 30+1 / 58_{2}+1 / 87_{3}+1 / 145_{5}$ \\
\hline 14 & 29 & 15 & 9 & 5 & 4 & 45 & $2 / 61=1 / 45+1 / 183_{3}+1 / 305_{5}+1 / 549_{9}$ \\
\hline 17 & 35 & 27 & 6 & 2 & 4 & 54 & $2 / 73=1 / 54+1 / 146_{2}+1 / 6579+1 / 1971_{27}$ \\
\hline 15 & 31 & 15 & 10 & 6 & 4 & 60 & $2 / 89=1 / 60+1 / 356_{4}+1 / 534_{6}+1 / 890_{10} \mathrm{Eg}$ \\
\hline 9 & 19 & 12 & 6 & 1 & 5 & 24 & $2 / 29=1 / 24+1 / 58_{2}+1 / 116_{4}+1 / 696_{24}$ \\
\hline 8 & 17 & 10 & 6 & 1 & 5 & 30 & $2 / 43=1 / 30+1 / 129_{3}+1 / 215_{5}+1 / 1290_{30}$ \\
\hline 11 & 23 & 14 & 7 & 2 & 5 & 42 & $2 / 61_{a}=1 / 42+1 / 183_{3}+1 / 366_{6}+1 / 1281_{21}$ \\
\hline 17 & 35 & 24 & 8 & 3 & 5 & 48 & $2 / 61_{b}=1 / 48+1 / 122_{2}+1 / 366_{6}+1 / 976_{16}$ \\
\hline 11 & 23 & 16 & 6 & 1 & 5 & 48 & $2 / 73_{\mathrm{a}}=1 / 48+1 / 219_{3}+1 / 584_{8}+1 / 3504_{48}$ \\
\hline 11 & 23 & 12 & 8 & 3 & 5 & 48 & $2 / 73_{b}=1 / 48+1 / 2924+1 / 4386+1 / 116816$ \\
\hline 12 & 25 & 18 & 6 & 1 & 5 & 54 & $2 / 83_{a}=1 / 54+1 / 249{ }_{3}+1 / 7479+1 / 448254$ \\
\hline 18 & 37 & 30 & 6 & 1 & 5 & 60 & $2 / 83_{b}=1 / 60+1 / 166_{2}+1 / 830_{10}+1 / 4980_{60}$ \\
\hline 11 & 23 & 14 & 7 & 2 & 5 & 56 & $2 / 89=1 / 56+1 / 356_{4}+1 / 712_{8}+1 / 2492_{28}$ \\
\hline 14 & 29 & 18 & 9 & 2 & 7 & 36 & $2 / 43=1 / 36+1 / 86_{2}+1 / 172_{4}+1 / 774_{18}$ \\
\hline 9 & 19 & 10 & 8 & 1 & 7 & 40 & $2 / 61=1 / 40+1 / 2444_{4}+1 / 305_{5}+1 / 244040$ \\
\hline 23 & 47 & 30 & 12 & 5 & 7 & 60 & $2 / 73=1 / 60+1 / 146_{2}+1 / 365_{5}+1 / 876_{12}$ \\
\hline 14 & 29 & 18 & 9 & 2 & 7 & 54 & $2 / 79=1 / 54+1 / 237_{3}+1 / 474_{6}+1 / 2133_{27}$ \\
\hline 18 & 37 & 20 & 12 & 5 & 7 & 60 & $2 / 83=1 / 60+1 / 249_{3}+1 / 415_{5}+1 / 996_{12}$ \\
\hline 11 & 23 & 14 & 8 & 1 & 7 & 56 & $2 / 89=1 / 56+1 / 356_{4}+1 / 6237+1 / 498456$ \\
\hline 20 & 41 & 21 & 14 & 6 & 8 & 42 & $2 / 43=1 / 42+1 / 86_{2}+1 / 129_{3}+1 / 301_{7}^{E g}$ \\
\hline 15 & 31 & 15 & 12 & 4 & 8 & 60 & $2 / 89=1 / 60+1 / 356_{4}+1 / 445_{5}+1 / 1335_{15}$ \\
\hline 21 & 43 & 26 & 13 & 4 & 9 & 52 & $2 / 61=1 / 52+1 / 122_{2}+1 / 244_{4}+1 / 793_{13}$ \\
\hline 20 & 41 & 30 & 10 & 1 & 9 & 60 & $2 / 79_{a}=1 / 60+1 / 158_{2}+1 / 474_{6}+1 / 4740_{60}$ \\
\hline 20 & 41 & 20 & 15 & 6 & 9 & 60 & $2 / 79_{\mathrm{b}}=1 / 60+1 / 237_{3}+1 / 316_{4}+1 / 790_{10}{ }^{E g}$ \\
\hline 15 & 31 & 20 & 10 & 1 & 9 & 60 & $2 / 89=1 / 60+1 / 267_{3}+1 / 534_{6}+1 / 5340_{60}$ \\
\hline 25 & 51 & 35 & 14 & 2 & 12 & 70 & $2 / 89=1 / 70+1 / 178_{2}+1 / 445_{5}+1 / 3115_{35}$ \\
\hline 23 & 47 & 30 & 15 & 2 & 13 & 60 & $2 / 73=1 / 60+1 / 146_{2}+1 / 2924+1 / 219030$ \\
\hline 18 & 37 & 20 & 15 & 2 & 13 & 60 & $2 / 83=1 / 60+1 / 2493+1 / 3324+1 / 249030$ \\
\hline 24 & 49 & 32 & 16 & 1 & 15 & 64 & $2 / 79=1 / 64+1 / 158_{2}+1 / 316_{4}+1 / 5056_{64}$ \\
\hline 26 & 53 & 34 & 17 & 2 & 15 & 68 & $2 / 83=1 / 68+1 / 166_{2}+1 / 3324+1 / 282234$ \\
\hline 23 & 47 & 27 & 18 & 2 & 16 & 54 & $2 / 61=1 / 54+1 / 122_{2}+1 / 183_{3}+1 / 164727$ \\
\hline 27 & 55 & 36 & 18 & 1 & 17 & 72 & $2 / 89=1 / 72+1 / 178_{2}+1 / 356_{4}+1 / 640872$ \\
\hline 30 & 61 & 36 & 24 & 1 & 23 & 72 & $2 / 83=1 / 72+1 / 166_{2}+1 / 249_{3}+1 / 5976_{72}$ \\
\hline 33 & 67 & 39 & 26 & 2 & 24 & 78 & $2 / 89=1 / 78+1 / 178_{2}+1 / 267_{3}+1 / 3471_{39}$ \\
\hline
\end{tabular}

Table 14 shown above is only as an indication for us and, certainly, was not calculated in its entirety. 2/23 has been reported only for memory because it was solved at the end of Sect. 5.

With their experience related to 3-terms series, cut-off beyond 10 has been applied by the scribes. Indeed all cases (here 7) may support this cut-off without any exception.

Table 14 becomes Table 15 as shown below:

Table 15. [4-terms] options.

\begin{tabular}{|c|c|c|c|c|c|c|c|}
\hline n & $2 n+1$ & $d_{2}$ & $d_{3}$ & $d_{4}$ & $\Delta_{\mathrm{d}}$ & $D^{\mathrm{n}}{ }_{1}$ & Possible [4-terms] decompositions $\mathrm{m} 4 \leq 10$ \\
\hline 9 & 19 & 12 & 4 & 3 & 1 & 24 & $2 / 29=1 / 24+1 / 58_{2}+1 / 174_{6}+1 / 232_{8}^{E g}$ \\
\hline 9 & 19 & 10 & 5 & 4 & 1 & 40 & $2 / 61=1 / 40+1 / 244_{4}+1 / 488_{8}+1 / 610_{10} E g$ \\
\hline 14 & 29 & 14 & 8 & 7 & 1 & 56 & $2 / 83=1 / 56+1 / 332_{4}+1 / 581_{7}+1 / 664_{8}$ \\
\hline 5 & 11 & 5 & 4 & 2 & 2 & 20 & $2 / 29=1 / 20+1 / 116_{4}+1 / 145_{5}+1 / 29010$ \\
\hline 18 & 37 & 15 & 12 & 1 & 2 & 60 & $2 / 83=1 / 60+1 / 3324+1 / 4155+1 / 4986_{6}^{E g^{*}}$ \\
\hline 18 & 37 & 21 & 9 & 7 & 2 & 63 & $2 / 89=1 / 63+1 / 2673+1 / 623_{7}+1 / 801_{9}$ \\
\hline 8 & 17 & 10 & 5 & 2 & 3 & 20 & $2 / 23=1 / 20+1 / 46_{2}+1 / 92_{4}+1 / 230_{10}$ \\
\hline 23 & 47 & 20 & 15 & 12 & 3 & 60 & $2 / 73=1 / 60+1 / 219_{3}+1 / 2924+1 / 365_{5}{ }^{E g^{*}}$ \\
\hline 15 & 31 & 15 & 10 & 6 & 4 & 30 & $2 / 29=1 / 30+1 / 58_{2}+1 / 87_{3}+1 / 145_{5}$ \\
\hline
\end{tabular}




\begin{tabular}{|c|c|c|c|c|c|c|c|}
\hline n & $2 n+1$ & $\mathbf{d}_{2}$ & $d_{3}$ & $\mathbf{d}_{4}$ & $\Delta^{\prime}{ }_{d}$ & $D^{n_{1}}$ & Possible [4-terms] decompositions $\mathrm{m} 4 \leq \mathbf{1 0}$ \\
\hline 14 & 29 & 15 & 9 & 5 & 4 & 45 & $2 / 61=1 / 45+1 / 183_{3}+1 / 305_{5}+1 / 549_{9}$ \\
\hline 15 & 31 & 15 & 10 & 6 & 4 & 60 & $2 / 89=1 / 60+1 / 356_{4}+1 / 534_{6}+1 / 890_{10} E g$ \\
\hline 20 & 41 & 21 & 14 & 6 & 8 & 42 & $2 / 43=1 / 42+1 / 86_{2}+1 / 129_{3}+1 / 301{ }_{7}^{E g}$ \\
\hline 20 & 41 & 20 & 15 & 6 & 9 & 60 & $2 / 79=1 / 60+1 / 237_{3}+1 / 316_{4}+1 / 790_{10} E g$ \\
\hline
\end{tabular}

We follow the same way as for the [3-terms] series with slightly different subsets. That yields:

Table 16. A single or two different $\triangle{ }^{\prime}{ }_{d}$ [4-terms].

\begin{tabular}{|c|c|c|c|c|c|c|c|}
\hline \multicolumn{7}{|c|}{ D with a single $\Delta^{\prime}{ }_{d}$ (options: no) } & \multirow{2}{*}{$\begin{array}{l}\text { Scribes's decision: obvious } \\
\text { [4-terms] decompositions } \\
\end{array}$} \\
\hline $\mathbf{n}$ & $2 n+1$ & $\mathbf{d}_{2}$ & $d_{3}$ & $d_{4}$ & $\Delta_{\mathbf{d}_{\mathrm{d}}}^{\prime}$ & $D^{\mathrm{n}}{ }_{1}$ & \\
\hline 23 & 47 & 20 & 15 & 12 & 3 & 60 & $2 / 73=1 / 60+1 / 219_{3}+1 / 292_{4}+1 / 365_{5}{ }^{E g^{*}}$ \\
\hline 20 & 41 & 21 & 14 & 6 & 8 & 42 & $2 / 43=1 / 42+1 / 86_{2}+1 / 129_{3}+1 / 301_{7}^{E g}$ \\
\hline 20 & 41 & 20 & 15 & 6 & 9 & 60 & $2 / 79=1 / 60+1 / 237_{3}+1 / 316_{4}+1 / 790_{10}{ }^{E g}$ \\
\hline
\end{tabular}

\begin{tabular}{|c|c|c|c|c|c|c|c|}
\hline \multicolumn{7}{|c|}{ D with two different $\Delta^{\prime}{ }_{\mathrm{d}}$ (options: yes) } & \multirow{2}{*}{$\begin{array}{l}\text { Scribes's decision: smallest } \Delta_{d}^{\prime} \\
\text { [4-terms] decompositions }\end{array}$} \\
\hline n & $2 n+1$ & $\mathbf{d}_{2}$ & $\mathbf{d}_{3}$ & $d_{4}$ & $\Delta_{\mathrm{d}}^{\prime}$ & $D^{n_{1}}$ & \\
\hline 9 & 19 & 12 & 4 & 3 & 1 & 24 & $2 / 29=1 / 24+1 / 58_{2}+1 / 174_{6}+1 / 232_{8} E g$ \\
\hline 5 & 11 & 5 & 4 & 2 & 2 & 20 & $2 / 29=1 / 20+1 / 116_{4}+1 / 145_{5}+1 / 290_{10}$ \\
\hline 15 & 21 & 15 & 10 & 6 & 4 & 30 & $2 / 29=1 / 30+1 / 58_{2}+1 / 87_{3}+1 / 145_{5}$ \\
\hline 9 & 19 & 10 & 5 & 4 & 1 & 40 & $2 / 61=1 / 40+1 / 244_{4}+1 / 488_{8}+1 / 610_{10}{ }^{E g}$ \\
\hline 14 & 29 & 15 & 9 & 5 & 4 & 45 & $2 / 61=1 / 45+1 / 183_{3}+1 / 305_{5}+1 / 549_{9}$ \\
\hline
\end{tabular}

\begin{tabular}{llllllll}
\hline & & & & & Scribes's decision: consecutive multipliers \\
\hline $\mathbf{n}$ & $\mathbf{2 n}+\mathbf{1}$ & $\mathbf{d}_{\mathbf{2}}$ & $\mathbf{d}_{\mathbf{3}}$ & $\mathbf{d}_{\mathbf{4}}$ & $\boldsymbol{\Delta}_{\mathbf{d}}^{\mathbf{d}_{\mathbf{1}}}$ & $\boldsymbol{D}^{\mathbf{n}} \mathbf{1}_{\mathbf{1}}$ & [4-terms] decompositions \\
\hline 14 & 29 & 14 & 8 & 7 & 1 & 56 & $2 / 83=1 / 56+1 / 332_{4}+1 / 581_{7}+1 / 664_{8}$ \\
18 & 37 & 15 & 12 & 10 & 2 & 60 & $2 / 83=1 / 60+1 / 332_{4}+1 / 415_{5}+1 / 498_{6}{ }^{E g^{*}}$ \\
\hline
\end{tabular}

\begin{tabular}{llllllll}
\hline & & & & & \multicolumn{3}{c}{ Scribes's decision: no odd denominator $\boldsymbol{D}_{\boldsymbol{1}}$} \\
\hline $\mathbf{n}$ & $\mathbf{2 n + 1}$ & $\mathbf{d}_{\mathbf{2}}$ & $\mathbf{d}_{\mathbf{3}}$ & $\mathbf{d}_{\mathbf{4}}$ & $\boldsymbol{\Delta}_{\mathbf{d}}$ & $\boldsymbol{D}_{\mathbf{1}}^{\mathbf{n}_{\mathbf{1}}}$ & {$[$ 4-terms] decompositions } \\
\hline 18 & 37 & 21 & 9 & 7 & 2 & 63 & $2 / 89=1 / 63+1 / 267_{3}+1 / 623_{7}+1 / 801_{9}$ \\
15 & 31 & 15 & 10 & 6 & 4 & 60 & $2 / 89=1 / 60+1 / 356_{4}+1 / 534_{6}+1 / 890_{10}{ }_{E g}$ \\
\hline
\end{tabular}

We recall that any odd denominator $D_{l}$ could lead to a solution for [3-terms] decompositions as checked in tables 4 or 5. Its occurrence arises only 2 times in table 12 [4-terms]. The first, for $2 / 61$, was dropped out because a $\Delta^{\prime}{ }_{\mathrm{d}}=4$ too high. The second one regards 2/89 (first row). Then, for a unifying sake, avoiding singularity, chief scribe decided to discard $D_{1}=63$ in this case.

Remark that we are very far from assumptions of Gillings [4] about Egyptian preferences for even numbers instead of odd, regarding the denominators in general. Thus the "no odd precept' was a low priority. At low ratio also (2 times only), this will be applied to the composite numbers $D$ [12].

\section{Conclusion}

As we saw, the most recent analysis (2008) has been performed on the ' $2 / \mathrm{n}$ ' table by Abdulaziz [10] (see his group $G_{2}$ ). It can be appreciated as a kind of mathematical anastylosis using materials issued from the RMP and other documentation. Ancient calculation procedure, using mainly fractions, is faithfully respected, but leads to arithmetical depth analyses of each divisor of $D_{l}$.

Our global approach avoided the difficulties of sophisticated arithmetical studies. This provides the advantage of forgetting quickly some widespread 'modern' ideas about the topic.

- No, the last denominator is not bounded by a fixed value of 1000. It only depends on the 'circumstances' related to the value of $D$. For 3 or 4 terms, a limitation like $D_{h} \leq$ $10 D$ is quite suitable, except only for $2 / 53$ where 10 is replaced by 15 . An observation well stressed in Ref. [10].

- No requirement is found about the denominator $D_{l}$ as having to be the greatest if alternatives.

- Once for all, a systematic predilection for even denominators does not need to be considered. Only once, we were forced to discard $D_{1}=63$ (odd) for deciding on $2 / 89$.

- Of course, there is no theoretical formula that can give immediately the first denominator as a function of $D$. It must necessarily go through trials and few selection criteria. The simpler the better, like the $\Delta$-classification presented in this paper. Maybe is it this classification that induces the opportunity of a comprehensive approach ? Strictly speaking, there are no algorithms in the method, just tables and pertinent observation. This is how $2 / 23$, $2 / 29$ or $2 / 53$ have found a logical explanation, more thorough than the arguments commonly supplied for these 'singularities'.

Find a simple logic according to which there is no singular 
case was the goal of the present paper.

Perhaps, chronologically, the study of prime numbers has been elaborated before that of composite numbers. It is nothing more than an hypothesis consistent with the spirit of our study. Yes ancient scribes certainly have been able to calculate and analyze all the preliminary cases. Ultimately, our unconventional method allows us to reconstruct the table fairly easily with weak mathematical assumptions, except maybe the new idea to consider as beneficial to have consecutive multipliers.

Although we have not studied the case of $2 / 101$, it is interesting to see that there are 2 consecutive multipliers, since the Egyptian table provides

$$
2 / 101=1 / 101+1 / 202_{2}+1 / 303_{3}+1 / 606_{6} .
$$

\section{Appendix}

Why a boundary with a Top-flag?

In this appendix, we continue to consider prime denominators $D$. For [2-terms] decompositions this concept of a Top-flag has no meaning since the last denominator is unique.

Obviously, doubtless far from Egyptian concepts, there are another equations more general than (5) or (6), namely

$$
\begin{gathered}
2 / D=1 / D_{1}+1 / m_{2} D+1 / m_{3} D, \\
2 / D=1 / D_{1}+1 / m_{2} D+1 / m_{3} D+1 / m_{4} D .
\end{gathered}
$$

We can imagine these as issued from another kind of unity decomposition like

$$
\begin{gathered}
1=D / 2 D_{1}+1 / 2 m_{2}+1 / 2 m_{3}, \\
1=D / 2 D_{l}+1 / 2 m_{2}+1 / 2 m_{3}+1 / 2 m_{4} .
\end{gathered}
$$

$D / 2 D_{1}$ remains in the lead of equality and 1 is a sum of terms, each with a even denominator.

These (modern) equations have additional solutions of no use for the scribes.

A priori the solutions are infinite, then for avoiding such a tedious research (today and in the past time), it is necessary to limit the highest denominator $D_{h}=m_{h} D$. How to do that? Simply by defining a kind of 'Top-flag' $\mathrm{T}_{f}{ }^{[h]}$ such as

$$
D_{h} \leq D \mathbf{T}_{f}{ }^{[h]} \text {. }
$$

Indeed, as soon as one decides to study a three-terms decomposition or more, it should be realized that an upper boundary for the last denominator has to be fixed. If not, the number of solutions becomes infinite [countable]. Let us recall that $m_{2}<m_{3}<m_{4}$ and $D_{2}<D_{3}<D_{4}$. Unfortunately (or not) the author of this paper has begun the calculations with a even more general problem, this of solving

$2 / D=\sum_{i=1}^{h} 1 / D_{i}$ without any criteria of multiplicity involving multipliers like $m_{i}$ (i>2).

Certainly this was the reflex of Gillings [4] or Bruckheimer and Salomon [5]. The problem is solvable and the solutions available by means of a small computer. After a necessary arithmetical analysis, it can be found that $(h-1)$ sets of solutions exist. One with $(h-1)$ multipliers, another with $(h-2)$ multipliers and so on. No solution exists if one searches for $D_{i}$ $(i \geq 2)$ not multiple of $D$.

Even a low-level programming code like 'small basic' can be used instead of 'Fortran' to perform computations in a very acceptable speed. We quickly realized the necessity of stopping the calculations by using a limitation regarding the last highest denominator $D_{h}$. Whence the introduction of a Top-flag.

Actually the Egyptian 2/D table shows a subset of more general solutions because the multipliers $m_{i}$ have a specific form involving $D_{l}$ and some of its divisors $d_{i}$. For example out of this subset, you can find an unexpected [4-terms] solution for $2 / 23$ with $\mathrm{T}_{f}{ }^{[h]}=10$, namely

$$
2 / 23=1 / 15+1 / 115_{5}+1 / 138_{6}+1 / 230_{10} .
$$

So, if we restrict ourself to retrieve Egyptian fractions given in the table, it naturally comes to mind to limit the highest denominator by an upper boundary: a convenient Top-flag.

Excepted the Babylonian system example in base 60, a numeration in base 10 is rather universal, because of our two hands with each 5 fingers. It is of common sense that the selection was generally

$\mathrm{T}_{f}{ }^{[h]}=10(=2 \times 5)$, not excluding a favorable appreciation for $\mathrm{T}_{f}^{[3]}=15(=3 \times 5)$ as for $2 / 53$.

\section{References}

[1] T. E. PEET: The Rhind Mathematical Papyrus, British Museum 10057 and 10058, London: The University Press of Liverpool limited and Hodder - Stoughton limited (1923).

[2] A. B. CHACE; 1. BULL; H. P. MANNING; and R. C. ARCHIBALD: The Rhind Mathematical Papyrus, Mathematical Association of America, Vol.1 (1927), Vol. 2 (1929), Oberlin, Ohio.

[3] G. ROBINS and C. SHUTE: The Rhind Mathematical Papyrus: An Ancient Egyptian Text, London: British Museum Publications Limited, (1987). [A recent overview].

[4] R. J. GILLINGS: Mathematics in the Time of Pharaohs, MIT Press (1972), reprinted by Dover Publications (1982).

[5] M. BRUCKHEIMER and Y. SALOMON: Some comments on R. J Gillings's analysis of the $2 / \mathrm{n}$ table in the Rhind Papyrus, Historia Mathematica, Vol. 4, pp. 445-452 (1977).

[6] E. R. ACHARYA: "Mathematics Hundred Years Before and Now", History Research, Vol. 3, No 3, pp. 41-47, (2015).

[7] K. R. W. ZAHRT: Thoughts on Ancient Egyptian Mathematics Vol. 3, pp. 90-93 (2000), [available as pdf on the Denver site https://scholarworks.iu.edu/journals/index.php/iusburj/.../19842]

[8] G. LEFEBVRE: In: Grammaire de L'Egyptien classique, Le Caire, Imprimerie de l'IFAO, 1954.

[9] A. IMHAUSEN and J. RITTER: Mathematical fragments [see fragment UC32159]. (2004). In: The UCL Lahun Papyri, Vol. 2, pp. 71-96. Archeopress, Oxford, Eds M. COLLIER, S. QUIRKE. 
[10] A. ABDULAZIZ: On the Egyptian method of decomposing 2/n into unit fractions, Historia Mathematica, Vol. 35, pp. 1-18 (2008).

[11] M. GARDNER: Egyptian fractions: Unit Fractions, Hekats and Wages - an Update (2013), available on the site of academia. edu. [Herein can be found an historic of various researches about the subject].
[12] L. BREHAMET: Remarks on the Egyptian 2/D table in favor of a global approach (D composite number), arXiv:1404.0341 [math. HO] (2014).

[13] L. FIBONACCI: Liber abaci (1202).

[14] E. M. BRUINS: The part in ancient Egyptian mathematics, Centaurus, Vol. 19, pp. 241-251 (1975). 